\title{
Secrecy Performance Analysis of Wireless Communications in the Presence of UAV Jammer and Randomly Located UAV Eavesdroppers
}

\author{
Jinchuan Tang, Student Member, IEEE, Gaojie Chen, Senior Member, IEEE, \\ and Justin P. Coon, Senior Member, IEEE
}

\begin{abstract}
Unmanned aerial vehicles (UAVs) have been undergoing fast development for providing broader signal coverage and more extensive surveillance capabilities in military and civilian applications. Due to the broadcast nature of the wireless signal and the openness of the space, UAV eavesdroppers (UEDs) pose a potential threat to ground communications. In this paper, we consider the communications of a legitimate ground link in the presence of friendly jamming and UEDs within a finite area of space. The spatial distribution of the UEDs obeying a uniform binomial point process (BPP) is used to characterize the randomness of the UEDs. The ground link is assumed to experience log-distance path loss and Rayleigh fading, while free space path loss with/without the averaged excess path loss due to the environment is used for the air-to-ground/air-to-air links. A piecewise function is proposed to approximate the line-of-sight (LoS) probability for the air-to-ground links, which provides a better approximation than using the existing sigmoid-based fitting. The analytical expression for the secure connection probability (SCP) of the legitimate ground link in the presence of non-colluding UEDs is derived. The analysis reveals some useful trends in the SCP as a function of the transmit signal to jamming power ratio, the locations of the UAV jammer, and the height of UAVs.
\end{abstract}

Index Terms-Unmanned aerial vehicles, physical layer security, secure connection probability, binomial point process, jamming, non-colluding eavesdroppers.

\section{INTRODUCTION}

$\mathbf{U}$ NMANNED aerial vehicles (UAVs), also known as drones, are a promising technology that offers reliable and cost-effective wireless communication solutions in a wide range of real-world scenarios [1]. Recently, the low-altitude UAVs with elevated height from hundreds of meters to several kilometers have drawn much research attention in surveillance, public safety and secure communications [2]-[4]. Compared to the existing terrestrial communication systems, UAV aided wireless networks have the potential to overcome the propagation constraints due to terrain characteristics, enhance the signal coverage and reduce operating cost [4]. Due to the broadcast nature of the wireless signal and the openness of the space, ground communications are susceptible to eavesdropping. UAV eavesdroppers (UEDs) could pose a greater threat to the security of ground communications than ground eavesdroppers, since UEDs are less constrained by terrain characteristics and a higher

This work was supported by the EPSRC through "Spatially Embedded Networks" under Grant EP/N002350/1.

J. Tang and J. P. Coon are with the Department of Engineering Science, University of Oxford, Oxford, UK e-mail: \{jinchuan.tang, justin.coon\}@eng.ox.ac.uk.

G. Chen is with the Department of Engineering, University of Leicester, Leicester, UK e-mail: gaojie.chen@leicester.ac.uk. chance of line-of-sight (LoS) link with stronger signal strength can be formed from the ground transmitter to the UEDs rather than to ground eavesdroppers. An early proof-of-concept called 'Snoopy drone' has already been demonstrated by hackers at the Black Hat security conference during 2014 in Singapore to carry a payload containing the equipment necessary to exploit security weaknesses in mobile phones and steal data. Furthermore, many aspects of UAVs, such as low production cost, high mobility, and ease of operation, could incentivize the attackers to use UAVs as the major eavesdropping tools. According to [5], spying UAVs have already caused privacy and security concerns to the public. Consequently, it has become increasingly urgent and necessary to study the security of ground-based wireless communication in the presence of UEDs.

\section{A. Motivation and related work}

Among the many methods for securing wireless communication, physical layer security (PLS) has emerged as a promising technique for achieving a secure transmission by exploiting the channel characteristics through signal processing techniques and channel coding without the need of a shared secret key [6], [7]. By using an information theoretic formalism, PLS has been shown to support perfect secrecy under realistic condition [8]. Due to the fading effects of wireless channels and the unpredictable locations of eavesdroppers, probabilistic approaches have been used to characterize the likelihood of a link achieving a secrecy rate, namely, secure connection probability (SCP) [9]. Stochastic geometry has been exploited for the analysis of eavesdropping wireless networks by endowing the locations of the eavesdroppers with a probability distribution, such as the Poisson point process (PPP) or the binomial point process (BPP) [10]-[12]. Meanwhile, the spectrum sharing of super dense drone small cell networks modeled by a 3D PPP was studied in [13]. The authors of [14] considered the case that the number of UAVs is small and deployed to cover a given finite region with a more suitable homogeneous BPP for UAV networks under Nakagami-m fading. Since it is common in deployment scenarios (especially in suburban and rural areas) to have a significantly stronger LoS component rather than reflected multipath components, the coverage probability in the absence of fading has been derived in [14]. Furthermore, to capture the performance of an air-to-ground (ATG) link between a ground device and a UAV, the channel propagation model incorporating blockages from buildings is required. Based on the statistical model for building blockages [15], the LoS probability in the product of a sequence of terms and its approximation via 
sigmoid function was formulated, and the optimal altitude for deploying a UAV with maximum coverage was studied in [16].

There have been a lot of studies on either optimizing network resources or developing techniques for realistic systemlevel analysis of UAV networks on coverage, but it has been pointed out by [4] that very few studies have investigated the secrecy performance of UAV networks, and only ground-based eavesdroppers were considered in those scenarios. In [17], the optimization of the secrecy rate of a UAV-enabled mobile relay system was formulated, and a performance gain over static relaying was achieved. By jointly designing the trajectory and transmit power of the UAV, an optimization algorithm designed to achieve the average worst-case secrecy rate improvement in UAV-to-ground communications was proposed in [18]. By considering the transmit power of both the ground user and UAV in the joint design with trajectory, the average secrecy rate over a finite horizon was maximized for both the UAVto-ground and ground-to-UAV communications in the UAV assisted 5G wireless network [19]. Additionally, the authors of [4] investigated the secrecy performance of UAV networks working in the millimeter wave band, where the UAVs can be used either for information transmission or jamming, and it was revealed that the average achievable secrecy rate does not change monotonically with an increasing proportion of UAV jammers. Recently, the authors of [20] considered the application of a UAV friendly jammer to improve the PLS of a legitimate link, where the location of the ground eavesdropper is unknown. Meanwhile, the authors of [21] proposed to employ a mobile UAV-enabled jammer to improve the secrecy rate of the ground wire-tap channel by moving towards and opportunistically jamming the discovered ground eavesdropper. UAV jamming is more appealing than ground jamming, because the air-to-air wireless channel from the UAV jammer to UEDs is in a pure LoS state with free space path loss, while the ground-to-air channel from a ground jammer to the UEDs would experience the additional excess path loss in the urban environment due to shadowing and scattering caused by the man-made structures [16]. Although it is known that increasing the jamming power will pose a stronger interference to both the UEDs and the legitimate ground receiver, how the secrecy performance behaves with respect to this increase is still unknown. In addition, according to [22], the demand for counter-drone systems based on UAV, handheld, and ground platforms have only emerged since the past few years to tackle the potential security threats posed by the UAVs. To fulfil the demand, it is vital to analyze the secrecy performance of the ground link against the randomly located UEDs by using the UAV jammer. Furthermore, the coverage and secrecy analysis in the previous literature has suffered from the tractability problem when the LoS probability is considered, since the analysis always results in multi-integral expressions.

\section{B. Contributions}

This paper focuses on the secrecy performance of groundbased communications in the presence of a UAV jammer and UEDs. The main contributions of the paper are summarized as follows:

1) LoS model: This is the first work to propose an approximation to simplify the modeling of the probability of LoS

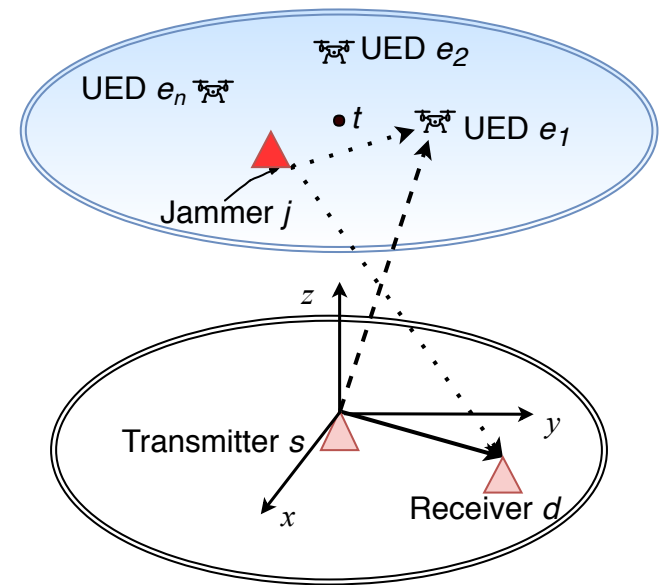

Fig. 1. The system model for communication in the presence of UEDs. The bold solid arrow denotes the legitimate link. For UED $e_{1}$, the dashed arrow denotes the wire-tap link, and the dotted arrows denote the jamming links.

channels, which allows us to get tractable formulations in the analysis of wireless networks concerning ATG channels. The trend of the LoS probability with respect to the height of UAVs has been captured in a simplistic manner.

2) UAV jammer: We introduce a UAV jammer for improving the security of the ground communication, and we give the cumulative distribution function (CDF) of the signal-to-interference ratio (SIR) from a ground transmitter to a UED subject to interference by the UAV jammer.

3) SCP: We formulate the SCP of the ground link in the presence of randomly located UEDs for the non-colluding scenario. The trends of the SCP in some environments with respect to different transmit signal to jamming power ratios, and the locations of the UAV jammer have been analysed.

The rest of the paper is structured as follows. Section II begins with a description of the system model then addresses the LoS probability and its approximations. Section III focuses on the derivation of the SCP. Section IV focuses on the behaviour of the SCP for different parameters. Section V gives the simulation results and discussion, and Section VI concludes the paper.

\section{SySTEM MOdeL}

\section{A. Network layout}

As shown in Fig. 1, transmitter $s$ at $(0,0,0) \in \mathbb{R}^{3}$ connects to receiver $d$ at $\left(x_{d}, y_{d}, 0\right)$ via the legitimate link, where $x_{d}$ and $y_{d}$ denotes the locations of $d$ on the $\mathrm{x}$-axis and $\mathrm{y}$-axis, respectively. There are $n$ UEDs uniformly distributed in a disk, forming a uniform BPP [14]. The disk is denoted as $O\left(t, R_{1}\right)$, where $O$ denotes the two-dimensional disk of radius $R_{1}$ centred at $t$. The coordinates of the origin of the disk are $(0,0, H)$ so that the center of the circular plane $t$ is $H$ meters right above the transmitter $s$ on the ground, and the disk is also parallel to the ground ${ }^{1}$. The UEDs work independently to decode the received signal on their links. Meanwhile, a UAV jammer $j$ is

\footnotetext{
${ }^{1}$ We assume that the ground between $s$ and $d$ is flat, and the effect due to the spherical surface of the earth is negligible.
} 
also located within the disk $O\left(t, R_{1}\right)$ and continually sends a jamming signal. The ground channel is assumed to experience Rayleigh fading and path loss, while the channel model for the ATG communications is based on the probabilistic LoS and non-line-of-sight (NLoS) links given by [16], [23]. The air-to-air communication channel is assumed to follow Friis free space transmission. The transmit powers of $s$ and $j$ are given by $P_{s}$ and $P_{j}$, respectively. The proposed system model can be applied to the application scenarios such as countersurveillance against the illegal amateur drones monitoring on ground radio communications and anti-eavesdropping against the compromised or untrustworthy UAV base stations in the future UAV-assisted communications systems.

\section{B. Channel model}

1) Air-to-air channel model: Since no major obstacles are obstructing the communications in the sky, the air-to-air channel follows Friis free space transmission. And the received jamming power from $j$ to $e$ is given by

$$
P_{j, e}=P_{j}(\lambda / 4 \pi)^{2} l_{j, e}^{-\alpha}
$$

where $\alpha=2$ is the free space path loss (FSPL) exponent, and $l_{x, y}$ is the distance between nodes $x$ and $y$.

2) ATG channel models: The potential existence of buildings and other obstructions lying in the propagation path results in the presence of mixed LoS/NLoS channel conditions between an air terminal and a ground terminal. According to [23], the ATG channel model will be mainly based on probabilistic LoS and NLoS links instead of following a classical fading channel. Therefore, the corresponding received signal powers at $e$ from transmitter $s$ for LoS and NLoS links are written as

$$
P_{s, e}= \begin{cases}\eta_{\mathrm{L}} P_{s}(\lambda / 4 \pi)^{2} l_{s, e}^{-\alpha}, & \text { LoS link } \\ \eta_{\mathrm{N}} P_{s}(\lambda / 4 \pi)^{2} l_{s, e}^{-\alpha}, & \text { NLoS link }\end{cases}
$$

and $\eta_{\mathrm{L}}$ and $\eta_{\mathrm{N}}$ refer to the mean values of excess path loss added to the FSPL, where $\left(\eta_{\mathrm{L}}, \eta_{\mathrm{N}}\right)$ can be measured at $f_{c}=2$ $\mathrm{GHz}$ in $\mathrm{dB}$ to be $(-0.1,-21),(-1.0,-20),(-1.6,-23)$, and $(-2.3,-34)$ for suburban, urban, dense urban, and highrise urban areas, respectively [16].

a) LoS probability and sigmoid fitting: The probabilities for a link $(s, e)$ to be either LoS or NLoS can be denoted as $\mathbb{P}_{\mathrm{L}}^{s, e}$ and $\mathbb{P}_{\mathrm{N}}^{s, e}=1-\mathbb{P}_{\mathrm{L}}^{s, e}$, respectively. According to [15], [16],

$$
\begin{aligned}
\mathbb{P}_{\mathrm{L}}^{s, e} & =\prod_{l=0}^{f(r)}\left[1-\exp \left(-\frac{(H-(l+1 / 2) H /(f(r)+1))^{2}}{2 \sigma^{2}}\right)\right] \\
& \approx \frac{1}{1+C \exp \left(-B\left(\theta_{s, e}-C\right)\right)}
\end{aligned}
$$

where $f(r)=\left\lfloor r \sqrt{\rho_{1} \rho_{2}}-1\right\rfloor, r=l_{t, e}$ is the ground distance between $s$ and $e, \rho_{1}$ is the ratio of built-up land area to the total land area, $\rho_{2}$ is the mean number of buildings per unit area (buildings $/ \mathrm{km}^{2}$ ), and $\sigma$ is the scale parameter of the Rayleigh probability density function (PDF), which gives the height distribution of buildings in meters. The environment parameters $\left(\rho_{1}, \rho_{2}, \sigma\right)$ for typical environments are suburban $(0.1,750,8)$, urban $(0.3,500,15)$, dense urban $(0.5,300,20)$, and highrise urban $(0.5,300,50)$, respectively. Note that increasing $H$ will

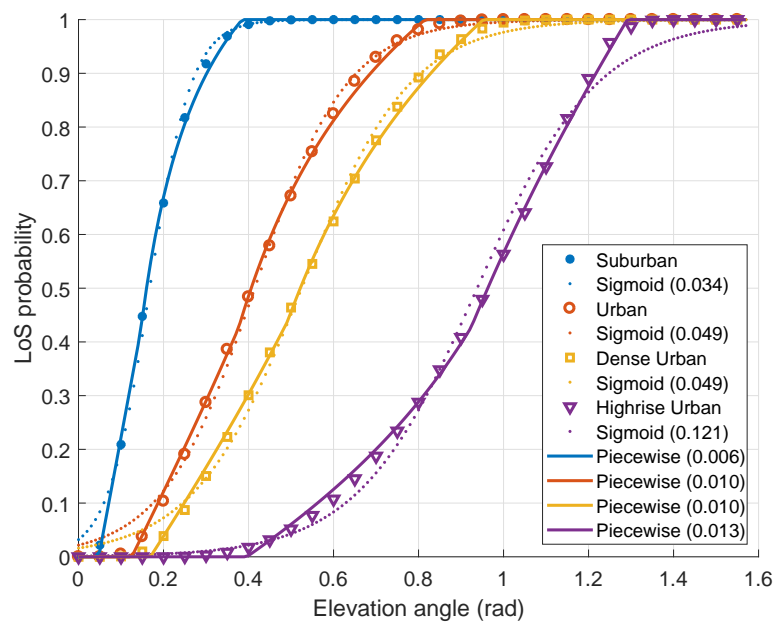

Fig. 2. Plots of the LoS probabilities for different urban environments based on the exact product, the sigmoid fitting and the proposed piecewise fitting. The goodness of fit measure by root-mean-square error is given inside the brackets.

increase the smoothness of the plot of the product, and $\mathbb{P}_{\mathrm{L}}^{s, e}$ can be considered as a continuous function of elevation angle and the environment parameters for a large $H$ [16]. The approximation to the LoS probability of an ATG link is given by a sigmoid function, where $C$ and $B$ are constant values depending on the aforementioned environment and $\theta_{s, e}$ is the elevation angle in radians, which is given by $\theta_{s, e}=\tan ^{-1}\left(\frac{H}{l_{s, e}}\right)$. The ATG channel model also applies to the received jamming power $P_{j, d}$ with LoS and NLoS probability given by $\mathbb{P}_{\mathrm{L}}^{j, d}$ and $\mathbb{P}_{\mathrm{N}}^{j, d}$.

b) Proposed piecewise fitting: Although the sigmoid fitting provides a reasonably good approximation to the actual LoS probability, it provides a non-linear relationship to $l_{s, e}$ and has been shown to only give analytical forms which are non-trivial to evaluate. Based on the value of the elevation angle, each curve of the LoS probability can be divided into three approximated regions: LoS region for the link in a pure LoS state with high elevation angle, NLoS region for the the link in a pure NLoS state with low elevation angle, and a transitional region where the link is in mixed LoS/NLoS states. The transitional region can be further divided into two regions based on the slope of the curve as the elevation angle increases. As a result, we propose a piecewise fitting model in (4), which not only gives a meaningful relationship between the LoS probability and $l_{s, e}$ but also provides a tractable way to calculate the SCP as we will show later. The piecewise approximation is given by

$$
\mathbb{P}_{\mathrm{L}}^{s, e}= \begin{cases}1, & \text { if } \theta_{s, e}>\tan ^{-1} \frac{H}{\ell_{1}} \\ c_{3} \cot \left(\theta_{s, e}\right)+c_{4}, & \text { if } \tan ^{-1} \frac{H}{\ell_{2}}<\theta_{s, e} \leq \tan ^{-1} \frac{H}{\ell_{1}} \\ 0, & \text { if } \theta_{s, e} \leq \tan ^{-1} \frac{H}{\ell_{3}} \\ c_{1} \tan \left(\theta_{s, e}\right)+c_{2}, & \text { otherwise }\end{cases}
$$

where $\ell_{1}=H \frac{1-c_{4}}{c_{3}}, \ell_{2}=\frac{2 H c_{1}}{c_{4}-c_{2}-\sqrt{\left(c_{4}-c_{2}\right)^{2}+4 c_{1} c_{3}}}, \ell_{3}=-H \frac{c_{1}}{c_{2}}$, $c_{1}, c_{2}, c_{3}$ and $c_{4}$ are given in Table I and obtained by Algorithm 1 , and the corresponding results for different typical environments are plotted in Fig. 2. 
Algorithm 1: Finding parameters $c_{1}, c_{2}, c_{3}$ and $c_{4}$ for link $(s, e)$ given environment parameters $\left(\rho_{1}, \rho_{2}, \sigma\right)$.

Given: Environment parameters $\left(\rho_{1}, \rho_{2}, \sigma\right)$, thresholds: $c_{l}=0.005, c_{m}=0.5$, and $c_{u}=0.995$.

1 Generate $10^{4}$ evenly spaced samples from $[0, \pi / 2]$, and obtain the corresponding samples of LoS probabilities with (3).

2 Create a new dataset $D_{t_{1}}$ from the samples of LoS probabilities whose values are bigger than $c_{l}$ and smaller than $c_{m}$.

3 Find the coefficients $c_{1}$ and $c_{2}$ of the model function $c_{1} \tan \left(\theta_{s, e}\right)+c_{2}$ by fitting the function to $D_{t_{1}}$ with the trust region reflective least squares algorithm.

4 Let $c_{1} \tan \left(\theta_{s, e}\right)+c_{2}=0$, the maximum elevation angle for having only the NLoS link is $\tan ^{-1}\left(-\frac{c_{2}}{c_{1}}\right)$.

5 Create a new dataset $D_{t_{2}}$ from the samples of LoS probabilities whose values are bigger than $c_{m}$ and smaller than $c_{u}$.

6 Find the coefficients $c_{3}$ and $c_{4}$ of the model function $c_{3} \cot \left(\theta_{s, e}\right)+c_{4}$ by fitting the function to $D_{t_{2}}$ with the trust region reflective least squares algorithm.

7 Let $c_{3} \cot \left(\theta_{s, e}\right)+c_{4}=1$, the minimum elevation angle for having only the LoS link is $\tan ^{-1}\left(\frac{c_{3}}{1-c_{4}}\right)$.

8 The elevation angle at the inflection point, where the LoS probability changes from being $c_{1} \tan \left(\theta_{s, e}\right)+c_{2}$ to $c_{3} \cot \left(\theta_{s, e}\right)+c_{4}$ is $\tan ^{-1}\left(\frac{c_{4}-c_{2}-\sqrt{\left(c_{4}-c_{2}\right)^{2}+4 c_{1} c_{3}}}{2 c_{1}}\right)$, is obtained by solving $c_{1} \tan \left(\theta_{s, e}\right)+c_{2}=c_{3} \cot \left(\theta_{s, e}\right)+c_{4}$, and then selecting the root which provides a better fit to the samples of LoS probabilities.

TABLE I

THE PARAMETERS FOR (4) AND (5).

\begin{tabular}{|l|c|c|c|c|}
\hline & Suburban & Urban & Dense urban & Highrise urban \\
\hline$c_{1}$ & 4.215 & 1.581 & 1.201 & 0.4717 \\
$c_{2}$ & -0.2007 & -0.1991 & -0.2051 & -0.1972 \\
$c_{3}$ & -0.1341 & -0.3618 & -0.4864 & -1.223 \\
$c_{4}$ & 1.331 & 1.341 & 1.346 & 1.351 \\
\hline
\end{tabular}

Since $\tan \left(\theta_{s, e}\right)=\frac{H}{l_{t, e}}$, (4) can be further written as

$$
\mathbb{P}_{\mathrm{L}}^{s, e}= \begin{cases}1, & \text { if } l_{t, e}<\ell_{1} \\ c_{3} l_{t, e} / H+c_{4}, & \text { if } \ell_{1} \leq l_{t, e}<\ell_{2} \\ c_{1} H / l_{t, e}+c_{2}, & \text { if } \ell_{2} \leq l_{t, e}<\ell_{3} \\ 0, & \text { otherwise. }\end{cases}
$$

In Fig. 2, the proposed piecewise fitting outperforms the sigmoid fitting in the goodness of fit measured by root-meansquare error. The former is at least four times better than the latter in the suburban environment and nine times better in the highrise urban environment. The transition region is further approximated by a linear function of the variable $l_{t, e}$ and another linear function of the reciprocal of $l_{t, e}$. In the transition region, $\mathbb{P}_{\mathrm{L}}^{s, e}$ increases as $l_{t, e}$ decreases.

3) Ground-to-ground channel model: For ground communications, the log-distance path loss model is used to characterize the legitimate link from $s$ to $d$. The received power at $d$ from $s$ is written as

$$
P_{s, d}=P_{s}(\lambda / 4 \pi)^{2} l_{s, d}^{-\beta}\left|h_{s, d}\right|^{2}
$$

where $\lambda$ is the carrier wavelength, $\left|h_{s, d}\right|^{2}$ is the exponentially distributed channel power gain with unit mean [24, Equation (3.18)], and $\beta$ is the path loss exponent for ground communications.

\section{DERIVATION OF THE SCP}

\section{A. Secrecy capacity}

Let $\Phi=\{1,2, \ldots, n\}$ denote the collection of $n$ UEDs. The secrecy capacity of a link is the difference between the capacity of the main link and the capacity achieved via a collection of wire-tap links. The general form is given as [25]

$$
\begin{aligned}
C_{s} & =\left[\log _{2}\left(1+\operatorname{SINR}_{s, d}\right)-\log _{2}\left(1+\max _{e \in \Phi}\left(\operatorname{SINR}_{s, e}\right)\right)\right]^{+} \\
& =\left[\log _{2}\left(\frac{1+\frac{P_{s, d}}{P_{j, d}+N_{0}}}{1+\max _{e \in \Phi}\left(\frac{P_{s, e}}{P_{j, e}+N_{e}}\right)}\right)\right]^{+} \\
& \geq\left[\log _{2}\left(\frac{\Gamma_{1}}{\max _{e \in \Phi} \Gamma_{2}}\right)\right]^{+}
\end{aligned}
$$

where $\mathrm{SINR}_{s, y}$ denotes the signal-to-interference-plus-noise ratio from $s$ to $y, N_{0}$ is the additive white Gaussian noise (AWGN) power at the ground receiver $d, N_{e}$ is the AWGN power at a UED, and $[x]^{+}$denotes $\max (0, x)$ [26]. ' $\geq$ ' holds if the receivers on the UEDs have the low-noise figure by using the thermoelectric cooling and new materials [27]-[29]. $\Gamma_{1}=1+\frac{P_{s, d}}{P_{j, d}+N_{0}}$ represents $1+\operatorname{SINR}_{s, d}$, and $\Gamma_{2}=1+\frac{P_{s, e}}{P_{j, e}}$ represents $1+\operatorname{SIR}$ from $s$ to $e$, where the interference is due to UAV jammer $j$.

\section{B. SCP formulation}

The SCP is defined as

$$
p_{c}=\mathbb{P}\left(C_{s}>\mathcal{R}_{t}\right) \geq \mathbb{P}\left(\frac{\Gamma_{1}}{\max _{e \in \Phi} \Gamma_{2}}>2^{\mathcal{R}_{t}}\right)
$$

where $\mathcal{R}_{t} \geq 0$ is the target secrecy rate. Besides, $p_{c}=$ $\mathbb{P}\left(C_{s}>\mathcal{R}_{t}\right) \approx \mathbb{P}\left(\frac{\Gamma_{1}}{\max _{e} \in \Gamma_{2}}>2^{\mathcal{R}_{t}}\right)$, when both $d$ and UEDs are working in an interference-limited environment as the result of a large jamming signal. To evaluate the above inequality, we require the following calculations.

Since $\Gamma_{1}$ is a random variable related to $\left|h_{s, d}\right|^{2}$, the CDF of $\Gamma_{1}$ can be written as

$$
\begin{aligned}
& F_{\Gamma_{1}}\left(\gamma_{1}\right)=\mathbb{P}\left(1+\frac{P_{s, d}}{P_{j, d}+N_{0}}<\gamma_{1}\right) \\
& =\mathbb{P}\left(\frac{\left|h_{s, d}\right|^{2}}{\Lambda\left(\eta_{\mathrm{L}}\right)}<\gamma_{1}-1\right) \mathbb{P}_{\mathrm{L}}^{j, d}+\mathbb{P}\left(\frac{\left|h_{s, d}\right|^{2}}{\Lambda\left(\eta_{\mathrm{N}}\right)}<\gamma_{1}-1\right) \mathbb{P}_{\mathrm{N}}^{j, d} \\
& =1-\exp \left(\left(1-\gamma_{1}\right) \Lambda\left(\eta_{\mathrm{L}}\right)\right) \mathbb{P}_{\mathrm{L}}^{j, d}-\exp \left(\left(1-\gamma_{1}\right) \Lambda\left(\eta_{\mathrm{N}}\right)\right) \mathbb{P}_{\mathrm{N}}^{j, d}
\end{aligned}
$$

where $\Lambda(\eta)=\frac{l_{s, d}^{\beta}}{P_{s}}\left(\eta l_{j, d}^{-\alpha} P_{j}+N_{0}(\lambda / 4 \pi)^{-2}\right), l_{j, d}^{2}=l_{t, j}^{2}+$ $l_{s, d}^{2}-2 l_{t, j} l_{s, d} \cos \left(\varphi_{j^{\prime}}-\varphi_{d}\right)+H^{2}, j^{\prime}$ denotes the projection of $j$ on the $x y$-plane. $\varphi_{j^{\prime}}$ and $\varphi_{d}$ denote the angles of $l_{s, j^{\prime}}$ 
and $l_{s, d}$ measured counterclockwise from the $\mathrm{x}$-axis. Thus, the corresponding PDF is given by $f_{\Gamma_{1}}\left(\gamma_{1}\right)=\frac{\mathrm{d}}{\mathrm{d} \gamma_{1}} F_{\Gamma_{1}}\left(\gamma_{1}\right)$ such that

$$
\begin{aligned}
f_{\Gamma_{1}}\left(\gamma_{1}\right)= & \Lambda\left(\eta_{\mathrm{L}}\right) \exp \left(\left(1-\gamma_{1}\right) \Lambda\left(\eta_{\mathrm{L}}\right)\right) \mathbb{P}_{\mathrm{L}}^{j, d}+ \\
& \Lambda\left(\eta_{\mathrm{N}}\right) \exp \left(\left(1-\gamma_{1}\right) \Lambda\left(\eta_{\mathrm{N}}\right)\right) \mathbb{P}_{\mathrm{N}}^{j, d}
\end{aligned}
$$

Let $e^{\prime}$ denote the projection of the node $e$ on the $x y$-plane, and $\phi=\varphi_{j^{\prime}}-\varphi_{e^{\prime}} . \Gamma_{2}$ is a random variable related to the position of one UED $e$, which is uniformly distributed on the disk $O\left(t, R_{1}\right)$. Thus, the $\mathrm{CDF}$ of $\Gamma_{2}$ is given by

$$
\begin{aligned}
F_{\Gamma_{2}}\left(\gamma_{2}\right) & =\mathbb{E}_{e}\left[F_{1}\left(\gamma_{2}, l_{t, e}, \phi\right)\right] \\
& =\frac{1}{\pi R_{1}^{2}} \int_{0}^{2 \pi} \int_{0}^{R_{1}} F_{1}\left(\gamma_{2}, l_{t, e}, \phi\right) l_{t, e} \mathrm{~d} l_{t, e} \mathrm{~d} \phi
\end{aligned}
$$

where

$$
\begin{gathered}
F_{1}\left(\gamma_{2}, l_{t, e}, \phi\right)=g\left(\gamma_{2}, \eta_{\mathrm{L}}, l_{t, e}, \phi\right) \mathbb{P}_{\mathrm{L}}^{s, e}+g\left(\gamma_{2}, \eta_{\mathrm{N}}, l_{t, e}, \phi\right) \mathbb{P}_{\mathrm{N}}^{s, e} \\
\text { and } g(\gamma, \eta, \ell, \phi)=\mathbb{1}\left(1+\frac{\left(H^{2}+\ell^{2}\right)^{\frac{-\alpha}{2}} \eta P_{s}}{\left(l_{t, j}^{2}+\ell^{2}-2 l_{t, j} \ell \cos \phi\right)^{\frac{-\alpha}{2}} P_{j}} \leq \gamma\right) .
\end{gathered}
$$

\section{Calculation of the SCP}

When $\mathbb{P}_{\mathrm{L}}^{s, e}$ is given by (3), it is hard to find a closed-form expression for (11). To make progress, we use (4) instead, which can yield a closed-form expression for (11) with any given $R_{1}$ and $H$. With the help of (4), we can divide the LoS probability into multiple regions, and calculate their contributions to $F_{\Gamma_{2}}\left(\gamma_{2}\right)$ independently. This decomposition technique to obtain (11) seems to be tedious, but necessary, because it leads to a tractable solution. Throughout the following discussion, we refer to a number of propositions, which will be presented in Section III-D.

For $R_{1}<\ell_{1}$, where $l_{1}$ is considered in (5), the ATG link between transmitter $s$ and UED $e$ is always in a pure LoS state since $l_{t, e} \leq R_{1}$. Following Proposition 1, we have

$$
\begin{aligned}
F_{\Gamma_{2}}\left(\gamma_{2}\right) & =\int_{0}^{2 \pi} \int_{0}^{R_{1}} g\left(\gamma_{2}, \eta_{\mathrm{L}}, l_{t, e}, \phi\right) \frac{l_{t, e}}{\pi R_{1}^{2}} \mathrm{~d} l_{t, e} \mathrm{~d} \phi \\
& =F_{\Gamma_{3}}\left(\gamma_{2}, \eta_{\mathrm{L}}, R_{1}\right) .
\end{aligned}
$$

For $\ell_{1} \leq R_{1}<\ell_{2}$, the ATG link between transmitter $s$ and UED $e$ is in a pure LoS state if $l_{t, e}<\ell_{1}$, and the ATG link between transmitter $s$ and UED $e$ is in the mixed LoS/NLoS states with $\mathbb{P}_{\mathrm{L}}^{s, e}=c_{3} l_{t, e} / H+c_{4}$ if $\ell_{1} \leq l_{t, e} \leq R_{1}$. Following Propositions 1 and 3, we have

$$
\begin{aligned}
& F_{\Gamma_{2}}\left(\gamma_{2}\right)=\int_{0}^{2 \pi} \int_{0}^{\ell_{1}} g\left(\gamma_{2}, \eta_{\mathrm{L}}, l_{t, e}, \phi\right) \frac{l_{t, e}}{\pi R_{1}^{2}} \mathrm{~d} l_{t, e} \mathrm{~d} \phi+ \\
& \int_{0}^{2 \pi} \int_{\ell_{1}}^{R_{1}} F_{1}\left(\gamma_{2}, l_{t, e}, \phi\right) \frac{l_{t, e}}{\pi R_{1}^{2}} \mathrm{~d} l_{t, e} \mathrm{~d} \phi \\
& =F_{\Gamma_{3}}\left(\gamma_{2}, \eta_{\mathrm{L}}, \ell_{1}\right)+F_{\Gamma_{5}}\left(\gamma_{2}, \eta_{\mathrm{L}}, \eta_{\mathrm{N}}, R_{1}\right)-F_{\Gamma_{5}}\left(\gamma_{2}, \eta_{\mathrm{L}}, \eta_{\mathrm{N}}, \ell_{1}\right) .
\end{aligned}
$$

For $\ell_{2} \leq R_{1}<\ell_{3}$, the ATG link between transmitter $s$ and UED $e$ is in a pure LoS state if $l_{t, e}<\ell_{1}$, the ATG link between transmitter $s$ and UED $e$ is in the mixed LoS/NLoS states with $\mathbb{P}_{\mathrm{L}}^{s, e}=c_{3} l_{t, e} / H+c_{4}$ if $\ell_{1} \leq l_{t, e}<\ell_{2}$, and the ATG link between transmitter $s$ and UED $e$ is in the mixed LoS/NLoS states with $\mathbb{P}_{\mathrm{L}}^{s, e}=c_{1} H / l_{t, e}+c_{2}$ if $\ell_{2} \leq l_{t, e} \leq R_{1}$. Following Propositions 1, 2 and 3, we have

$$
\begin{aligned}
& F_{\Gamma_{2}}\left(\gamma_{2}\right)=\int_{0}^{2 \pi} \int_{0}^{\ell_{1}} g\left(\gamma_{2}, \eta_{\mathrm{L}}, l_{t, e}, \phi\right) \frac{l_{t, e}}{\pi R_{1}^{2}} \mathrm{~d} l_{t, e} \mathrm{~d} \phi+ \\
& \int_{0}^{2 \pi} \int_{\ell_{1}}^{R_{1}} F_{1}\left(\gamma_{2}, l_{t, e}, \phi\right) \frac{l_{t, e}}{\pi R_{1}^{2}} \mathrm{~d} l_{t, e} \mathrm{~d} \phi \\
= & F_{\Gamma_{3}}\left(\gamma_{2}, \eta_{\mathrm{L}}, \ell_{1}\right)+F_{\Gamma_{5}}\left(\gamma_{2}, \eta_{\mathrm{L}}, \eta_{\mathrm{N}}, \ell_{2}\right)-F_{\Gamma_{5}}\left(\gamma_{2}, \eta_{\mathrm{L}}, \eta_{\mathrm{N}}, \ell_{1}\right)+ \\
& F_{\Gamma_{4}}\left(\gamma_{2}, \eta_{\mathrm{L}}, \eta_{\mathrm{N}}, R_{1}\right)-F_{\Gamma_{4}}\left(\gamma_{2}, \eta_{\mathrm{L}}, \eta_{\mathrm{N}}, \ell_{2}\right) .
\end{aligned}
$$

For $R_{1} \geq \ell_{3}$, the ATG link between transmitter $s$ and UED $e$ is in a pure LoS state if $l_{t, e}<\ell_{1}$, the ATG link between transmitter $s$ and UED $e$ is in the mixed LoS/NLoS states with $\mathbb{P}_{\mathrm{L}}^{s, e}=c_{3} l_{t, e} / H+c_{4}$ if $\ell_{1} \leq l_{t, e}<\ell_{2}$, the ATG link between transmitter $s$ and UED $e$ is in the mixed LoS/NLoS states with $\mathbb{P}_{\mathrm{L}}^{s, e}=c_{1} H / l_{t, e}+c_{2}$ if $\ell_{2} \leq l_{t, e}<\ell_{3}$, and the ATG link between transmitter $s$ and UED $e$ is in a pure NLoS state if $\ell_{3} \leq l_{t, e} \leq R_{1}$. Following Propositions 1, 2 and 3, we have

$$
\begin{aligned}
& F_{\Gamma_{2}}\left(\gamma_{2}\right)=\int_{0}^{2 \pi} \int_{0}^{\ell_{1}} g\left(\gamma_{2}, \eta_{\mathrm{L}}, l_{t, e}, \phi\right) \frac{l_{t, e}}{\pi R_{1}^{2}} \mathrm{~d} l_{t, e} \mathrm{~d} \phi+ \\
& \int_{0}^{2 \pi} \int_{\ell_{1}}^{\ell_{3}} F_{1}\left(\gamma_{2}, l_{t, e}, \phi\right) \frac{l_{t, e}}{\pi R_{1}^{2}} \mathrm{~d} l_{t, e} \mathrm{~d} \phi+ \\
& \int_{0}^{2 \pi} \int_{\ell_{3}}^{R_{1}} g\left(\gamma_{2}, \eta_{\mathrm{N}}, l_{t, e}, \phi\right) \frac{l_{t, e}}{\pi R_{1}^{2}} \mathrm{~d} l_{t, e} \mathrm{~d} \phi \\
= & F_{\Gamma_{3}}\left(\gamma_{2}, \eta_{\mathrm{L}}, \ell_{1}\right)+F_{\Gamma_{5}}\left(\gamma_{2}, \eta_{\mathrm{L}}, \eta_{\mathrm{N}}, \ell_{2}\right)-F_{\Gamma_{5}}\left(\gamma_{2}, \eta_{\mathrm{L}}, \eta_{\mathrm{N}}, \ell_{1}\right)+ \\
& F_{\Gamma_{4}}\left(\gamma_{2}, \eta_{\mathrm{L}}, \eta_{\mathrm{N}}, \ell_{3}\right)-F_{\Gamma_{4}}\left(\gamma_{2}, \eta_{\mathrm{L}}, \eta_{\mathrm{N}}, \ell_{2}\right)+F_{\Gamma_{3}}\left(\gamma_{2}, \eta_{\mathrm{N}}, R_{1}\right)- \\
& F_{\Gamma_{3}}\left(\gamma_{2}, \eta_{\mathrm{N}}, \ell_{3}\right) .
\end{aligned}
$$

As a result of the above decomposition, $p_{c}$ is lower bounded by

$$
\mathbb{P}\left(\frac{\Gamma_{1}}{\max _{e \in \Phi} \Gamma_{2}}>2^{\mathcal{R}_{t}}\right)=\int_{1}^{\infty} F_{\Gamma_{2}}\left(\frac{\gamma_{1}}{2^{\mathcal{R}_{t}}}\right)^{n} f_{\Gamma_{1}}\left(\gamma_{1}\right) \mathrm{d} \gamma_{1}
$$

\section{Propositions}

All three propositions given below rely on Lemma 1, which is given in the Appendix.

Proposition 1. Let $r \leq R_{1}$, assuming that the link $(s, e)$ is always in the same channel state (i.e, pure LoS or pure NLoS) with gain $\eta$, the CDF of $\Gamma_{2}$ conditioning on that $e$ is only active for $l_{t, e} \leq r$ is given by

$$
F_{\Gamma_{3}}(y, \eta, r)=\int_{0}^{2 \pi} \int_{0}^{r} \frac{g\left(\gamma_{2}, \eta, l_{t, e}, \phi\right) l_{t, e}}{\pi R_{1}^{2}} \mathrm{~d} l_{t, e} \mathrm{~d} \phi=\frac{S_{1}(y, \eta, r)}{\pi R_{1}^{2} / 2}
$$

where

$$
S_{1}(y, \eta, r)= \begin{cases}D_{1}+D_{2}-\frac{1}{2} D_{3}, & \text { cases } 1,9,10 \\ D_{2}+D_{4}, & \text { cases } 2,7 \\ \frac{1}{2} \pi r^{2}-D_{3}, & \text { cases } 3,11 \\ D_{1}+D_{2}+\frac{1}{2} D_{3}, & \text { cases } 4,6,14 \\ \frac{1}{2} \pi r^{2}, & \text { cases } 5,12,13,19 \\ D_{3}, & \text { cases } 8,15 \\ D_{5}, & \text { cases 16, 17, } 18 \\ 0, & \text { otherwise }\end{cases}
$$


TABLE II

TABLE OF CONDITIONS.

\begin{tabular}{ll}
\hline Case index & Condition \\
\hline 1 & $A<0, B \leq 0, r^{2} A<B, \frac{-r^{2} A-B}{2^{2 r}} \leq l_{t, j}, l_{t, j} \neq \sqrt{B A}$ \\
2 & $A<0, B \leq 0, r^{2} A=B, \frac{-r^{2}-B}{2 r} \leq l_{t, j}, l_{t, j} \neq \sqrt{B A}$ \\
3 & $A<0, B \leq 0, r^{2} A<B, \sqrt{B A}<l_{t, j}<\frac{-r^{2} A-B}{2 r}$ \\
4 & $A<0, B \leq 0, r^{2} A>B, \frac{-r^{2} A-B}{2 r} \leq l_{t, j}$ \\
5 & Other cases under $A<0, B \leq 0$ \\
6 & $A>0, B \geq 0, r^{2} A>B, \frac{r^{2} A+B}{2 r} \leq l_{t, j}, l_{t, j} \neq \sqrt{B A}$ \\
7 & $A>0, B \geq 0, r^{2} A=B, \frac{r^{2} A+B}{2 r} \leq l_{t, j}, l_{t, j} \neq \sqrt{B A}$ \\
8 & $A>0, B \geq 0, r^{2} A>B, \sqrt{B A}<l_{t, j}<\frac{r^{2} A+B}{2 r}$ \\
9 & $A>0, B \geq 0, r^{2} A<B, \frac{r^{2} A+B}{2 r} \leq l_{t, j}$ \\
10 & $A<0, B>0, l_{t, j} \geq \frac{\left|r^{2} A+B\right|}{2 r}$ \\
11 & $A<0, B>0,-r^{2} A>B, l_{t, j}<\frac{-r^{2} A-B}{2 r}$ \\
12 & Other cases under $A<0, B>0$ \\
13 & $A>0, B<0,-r^{2} A>B, l_{t, j}<\frac{-r^{2} A-B}{2 r}$ \\
14 & $A>0, B<0, l_{t, j} \geq \frac{\left|r^{2} A+B\right|}{2 r}$ \\
15 & $A>0, B<0,-r^{2} A<B, l_{t, j}<\frac{r^{2} A+B}{2 r}$ \\
16 & $A=0, \frac{l_{t, j}^{2}-H^{2}}{{ }^{2}{ }^{2}} \leq l_{t, j}, l_{t, j}>H$ \\
17 & $A=0, \frac{H_{t, j}^{2}}{2 r} \leq l_{t, j}, l_{t, j}<H$ \\
18 & $A=0, l_{t, j}=H$ \\
19 & $A=0, l_{t, j}<\frac{H^{2}-l_{t, j}^{2}}{2 r}$ \\
\hline
\end{tabular}

and all the cases with indices are given in Table II, $D_{1}=$ $\frac{A B-l_{t, j}^{2}}{2 A^{2}} \cot ^{-1}\left(\frac{a_{1}-2 B}{\sqrt{4 l_{t, j}^{2} r^{2}-a_{1}^{2}}}\right), A=1-\left((y-1) \frac{P_{j}}{\eta P_{s}}\right)^{\frac{2}{\alpha}}$, $B=l_{t, j}^{2}-(1-A) H^{2}, a_{1}=A r^{2}+B, D_{2}=\frac{\pi r^{2}}{4}-$ $\frac{A}{4 A^{2}} \sqrt{4 l_{t, j}^{2} r^{2}-a_{1}^{2}}-\frac{a_{1} A-l_{t, j}^{2}}{2 A^{2}} \csc ^{-1}\left(\frac{2 l_{t, j} r}{a_{1}}\right), D_{3}=\frac{l_{t, j}^{2}-A B}{2 A^{2}} \pi$, $D_{4}=\frac{\left(a_{1}-2 B\right) \sqrt{4 j^{2} r^{2}-a_{1}^{2}}}{4 A^{2} r^{2}}, \quad D_{5}=\frac{1}{2} r^{2} \sec ^{-1}\left(\frac{2 l_{t, j} r}{a_{1}}\right)-$ $\frac{\left(l_{t, j}^{2}-H^{2}\right) \sqrt{4 l_{t, j}^{2} r^{2}-\left(H^{2}-l_{t, j}^{2}\right)^{2}}}{8 l_{t, j}^{2}}$.

Proof:

$$
\begin{aligned}
& \frac{1}{\pi R_{1}^{2}} \int_{0}^{2 \pi} \int_{0}^{r} g\left(y, \eta_{\mathrm{L}}, l_{t, e}, \phi\right) l_{t, e} \mathrm{~d} l_{t, e} \mathrm{~d} \phi \\
& =\frac{1}{\pi R_{1}^{2}} \int_{o_{\phi}(\eta)} \int_{o_{l_{t, e}}(\eta)} l_{t, e} \mathrm{~d} l_{t, e} \mathrm{~d} \phi \\
& =\frac{r^{2}}{R_{1}^{2}}-\frac{1}{\pi R_{1}^{2}} \int_{{o^{\prime}{ }_{\phi}(\eta)}} \int_{{o^{\prime}}_{l_{t, e}}(\eta)} l_{t, e} \mathrm{~d} l_{t, e} \mathrm{~d} \phi
\end{aligned}
$$

where $o_{\phi}(\eta)$ and $o_{l_{t, e}}(\eta)$ are the domains so that $1+$ $\frac{\left(H^{2}+l_{t, e}^{2}\right)^{\frac{-\alpha}{2}} \eta P_{s}}{\left(l_{t, j}^{2}+l_{t, e}^{2}-2 l_{t, j} l_{t, e} \cos \phi\right)^{\frac{-\alpha}{2}} P_{j}} \leq y$, while $o_{\phi}^{\prime}(\eta)$ and $o^{\prime}{ }_{t, e}(\eta)$ are the domains so that $1+\frac{\left(H^{2}+l_{t, e}^{2}\right)^{\frac{-\alpha}{2}} \eta P_{s}}{\left(l_{t, j}^{2}+l_{t, e}^{2}-2 l_{t, j} l_{t, e} \cos \phi\right)^{\frac{-\alpha}{2}} P_{j}}>y$. Given Lemma 1, (18) is achieved. Thus, we conclude the proof.

Proposition 2. Let $r \leq R_{1}$, assuming that the mixed LoS/NLoS states exist for link $(s, e)$, and the (quasi-) LoS probability is $\mathbb{P}_{\mathrm{L}}^{s, e}=c_{1} H / l_{t, e}+c_{2}$, the CDF of $\Gamma_{2}$ conditioning on the UED $e$ being active within the disk $O(t, r)$ is given by

$$
\begin{aligned}
F_{\Gamma_{4}}\left(y, \eta_{\mathrm{L}}, \eta_{\mathrm{N}}, r\right)= & \frac{2}{\pi R_{1}^{2}}\left(c_{2} S_{1}\left(y, \eta_{\mathrm{L}}, r\right)+c_{1} H S_{2}\left(y, \eta_{\mathrm{L}}, r\right)+\right. \\
& \left.\left(1-c_{2}\right) S_{1}\left(y, \eta_{\mathrm{N}}, r\right)-c_{1} H S_{2}\left(y, \eta_{\mathrm{N}}, r\right)\right)
\end{aligned}
$$

$$
S_{2}(y, \eta, r)= \begin{cases}G_{1}-G_{2}+G_{3}-G_{4}, & \text { cases } 1,2 \\ \pi r+G_{1}-2 G_{2}, & \text { cases } 3 \\ G_{3}+G_{4}-G_{2}, & \text { cases } 4,14 \\ \pi r, & \text { cases } 5,12,13,19 \\ G_{3}+G_{4}-G_{5}, & \text { cases } 6,7 \\ -G_{5}, & \text { case } 8 \\ G_{3}-G_{4}, & \text { cases } 9,10 \\ \pi r-G_{2}, & \text { case } 11 \\ -G_{2}, & \text { case } 15 \\ G_{6}, & \text { cases } 16,17 \\ \frac{1}{2} \pi r, & \text { case } 18 \\ 0, & \text { otherwise }\end{cases}
$$

and all the cases with indices are given in Table II, $G_{0}=\frac{2 \sqrt{-A B+l_{t, j}^{2}}}{-A}, G_{1}=G_{0} \mathrm{E}\left(\sec ^{-1}\left(\frac{-l_{t, j}}{\sqrt{B A}}\right) \mid \frac{l_{t, j}^{2}}{l_{t, j}^{2}-A B}\right)$, $G_{2}=G_{0} \mathrm{E}\left(\frac{l_{t, j}^{2}}{l_{t, j}^{2}-A B}\right), G_{3}=\frac{\sqrt{4 l_{t, j}^{2} r^{2}-a_{1}^{2}}-2 A r^{2} \sec ^{-1}\left(\frac{2 l_{t, j} r}{a_{1}}\right)}{-2 A r}$, $G_{4}=G_{0} \mathrm{E}\left(\sec ^{-1}\left(\frac{2 l_{t, j} r}{a_{1}}\right) \mid \frac{l_{t, j}^{2}}{l_{t, j}^{2}-A B}\right) / 2, \quad G_{5}=$ $G_{0} \mathrm{E}\left(\sec ^{-1}\left(\frac{l_{t, j}}{\sqrt{B A}}\right) \mid \frac{l_{t, j}^{2}}{l_{t, j}^{2}-A B}\right), G_{6}=r \cos ^{-1}\left(\frac{l_{t, j}^{2}-H^{2}}{2 l_{t, j} r}\right)-$ $\frac{l_{t, j}^{2}-H^{2}}{2 l_{t, j}} \ln \frac{\sqrt{4 l_{t, j}^{2} r^{2}-\left(H^{2}-l_{t, j}^{2}\right)^{2}}+2 l_{t, j} r}{\left|l_{t, j}^{2}-H^{2}\right|}, \mathrm{E}(\cdot)$ and $\mathrm{E}(\cdot \mid \cdot)$ denote complete and incomplete elliptic integral of the 2nd kind [30].

Proof:

$$
\begin{aligned}
\int_{0}^{2 \pi} & \int_{0}^{r}\left(g\left(y, \eta_{\mathrm{L}}, l_{t, e}, \phi\right) \mathbb{P}_{\mathrm{L}}^{s, e}+g\left(y, \eta_{\mathrm{N}}, l_{t, e}, \phi\right) \mathbb{P}_{\mathrm{N}}^{s, e}\right) l_{t, e} \mathrm{~d} l_{t, e} \mathrm{~d} \phi \\
= & \int_{o_{\phi}\left(\eta_{\mathrm{L}}\right)} \int_{o_{l_{t, e}}\left(\eta_{\mathrm{L}}\right)}\left(c_{1} H+c_{2} l_{t, e}\right) \mathrm{d} l_{t, e} \mathrm{~d} \phi+ \\
& \int_{o_{\phi}\left(\eta_{\mathrm{N}}\right)} \int_{o_{l_{t, e}}\left(\eta_{\mathrm{N}}\right)}\left(-c_{1} H+\left(1-c_{2}\right) l_{t, e}\right) \mathrm{d} l_{t, e} \mathrm{~d} \phi \\
= & c_{1} H \int_{o_{\phi}\left(\eta_{\mathrm{L}}\right)} \int_{o_{l_{t, e}}\left(\eta_{\mathrm{L}}\right)} \mathrm{d} l_{t, e} \mathrm{~d} \phi+2 c_{2} S_{1}\left(y, \eta_{\mathrm{L}}, r\right)- \\
& c_{1} H \int_{o_{\phi}\left(\eta_{\mathrm{N}}\right)} \int_{o_{l_{t, e}}\left(\eta_{\mathrm{N}}\right)} \mathrm{d} l_{t, e} \mathrm{~d} \phi+2\left(1-c_{2}\right) S_{1}\left(y, \eta_{\mathrm{N}}, r\right)
\end{aligned}
$$

where $\int_{o_{\phi}(\eta)} \int_{o_{l_{t, e}}(\eta)} \mathrm{d} l_{t, e} \mathrm{~d} \phi=2 \pi r-\int_{o_{\phi}^{\prime}(\eta)} \int_{{o^{\prime}}_{l_{t, e}}(\eta)} \mathrm{d} l_{t, e} \mathrm{~d} \phi$. Given the domains in Lemma 1 and dividing (23) by $\pi R_{1}^{2},(21)$ is achieved. Thus, we conclude the proof.

Proposition 3. Let $r \leq R_{1}$, assuming that the mixed LoS/NLoS states exist for link $(s, e)$, and the (quasi-) LoS probability is $\mathbb{P}_{\mathrm{L}}^{s, e}=c_{3} l_{t, e} / H+c_{4}$, the CDF of $\Gamma_{2}$ conditioning on the UED $e$ being active within the disk $O(t, r)$ is given by

$$
\begin{aligned}
F_{\Gamma_{5}}\left(y, \eta_{\mathrm{L}}, \eta_{\mathrm{N}}, r\right)= & \frac{2}{\pi R_{1}^{2}}\left(c_{4} S_{1}\left(y, \eta_{\mathrm{L}}, r\right)+\frac{c_{3}}{3 H} S_{3}\left(y, \eta_{\mathrm{L}}, r\right)+\right. \\
& \left.\left(1-c_{4}\right) S_{1}\left(y, \eta_{\mathrm{N}}, r\right)-\frac{c_{3}}{3 H} S_{3}\left(y, \eta_{\mathrm{N}}, r\right)\right)
\end{aligned}
$$


$S_{3}(y, \eta, r)= \begin{cases}M_{3}+M_{4}-M_{1}-M_{2}, & \text { cases } 1,2 \\ \pi r^{3}-2 M_{2}+M_{3}, & \text { case } 3 \\ M_{1}-M_{2}+M_{4}, & \text { cases } 4,14 \\ \pi r^{3}, & \text { cases } 5,12,13,19 \\ M_{1}-M_{3}+M_{4}, & \text { cases } 6,7 \\ -M_{3}, & \text { case } 8 \\ -M_{1}+M_{4}, & \text { cases } 9,10 \\ -M_{2}+\pi r^{3}, & \text { case } 11 \\ -M_{2}, & \text { case } 15 \\ \pi r^{3}-M_{5}-M_{6}, & \text { case } 16 \\ M_{5}+M_{6}, & \text { case } 17 \\ \pi r^{3} / 2, & \text { case } 18 \\ 0, & \text { otherwise }\end{cases}$

and all the cases with index numbers are given in Table II, $M_{0}=\frac{\sqrt{-A B+l_{t, j}^{2}}}{-3 A^{3} / 2}, \frac{2 M_{1}}{M_{0}}=4 B A \mathrm{~F}\left(\sec ^{-1}\left(\frac{l_{t, j} r}{a_{1} / 2}\right) \mid \frac{l_{t, j}^{2}}{l_{t, j}^{2}-A B}\right)+$ $\left(8 l_{t, j}^{2}-7 B A\right) \mathrm{E}\left(\sec ^{-1}\left(\frac{l_{t, j} r}{a_{1} / 2}\right) \mid \frac{l_{t, j}^{2}}{l_{t, j}^{2}-A B}\right), \quad M_{2}=$ $M_{0} 4 B A \mathrm{~K}\left(\frac{l_{t, j}^{2}}{l_{t, j}^{2}-A B}\right)+M_{0}\left(8 l_{t, j}^{2}-7 B A\right) \mathrm{E}\left(\frac{l_{t, j}^{2}}{l_{t, j}^{2}-A B}\right)$, $M_{3}=M_{0}\left(8 l_{t, j}^{2}-7 B A\right) \mathrm{E}\left(\sec ^{-1}\left(\frac{-l_{t, j}}{\sqrt{B A}}\right) \mid \frac{l_{t, j}^{2}}{l_{t, j}^{2}-A B}\right)+$ $M_{0} 4 B A \mathrm{~F}\left(\sec ^{-1}\left(\frac{-l_{t, j}}{\sqrt{B A}}\right) \mid \frac{l_{t, j}^{2}}{l_{t, j}^{2}-A B}\right), M_{4}=r^{3} \cos ^{-1} \frac{A r^{2}+B}{2 l_{t, j} r}+$ $\frac{M_{0}}{4 r}\left(A\left(2 a_{1}-9 B\right)+8 l_{t, j}^{2}\right) \sqrt{\frac{4 l_{t, j}^{2} r^{2}-a_{1}^{2}}{l_{t, j}^{2}-A B}}, \quad M_{5}=$ $r^{3} \cos ^{-1} \frac{\left|H^{2}-l_{t, j}^{2}\right|}{-2 l_{t, j} r}, M_{6}=\frac{r\left|H^{2}-l_{t, j}^{2}\right|}{8 l_{t, j}^{2}} \sqrt{4 l_{t, j}^{2} r^{2}-\left(H^{2}-l_{t, j}^{2}\right)^{2}}+$ $\frac{\left|H^{2}-l_{t, j}^{2}\right|^{3}}{16 l_{t, j}^{3}} \ln \frac{\sqrt{4 l_{t, j}^{2} r^{2}-\left(H^{2}-l_{t, j}^{2}\right)^{2}}+2 l_{t, j} r}{\left|H^{2}-l_{t, j}^{2}\right|}, \mathrm{K}(\cdot)$ and $\mathrm{F}(\cdot \mid \cdot)$ denote complete and incomplete elliptic integral of the 1st kind [30].

Proof:

$$
\begin{aligned}
\int_{0}^{2 \pi} & \int_{0}^{r}\left(g\left(y, \eta_{\mathrm{L}}, l_{t, e}, \phi\right) \mathbb{P}_{\mathrm{L}}^{s, e}+g\left(y, \eta_{\mathrm{N}}, l_{t, e}, \phi\right) \mathbb{P}_{\mathrm{N}}^{s, e}\right) l_{t, e} \mathrm{~d} l_{t, e} \mathrm{~d} \phi \\
= & \int_{o_{\phi}\left(\eta_{\mathrm{L}}\right)} \int_{o_{l_{t, e}}\left(\eta_{\mathrm{L}}\right)}\left(c_{3} l_{t, e}{ }^{2} / H+c_{4} l_{t, e}\right) \mathrm{d} l_{t, e} \mathrm{~d} \phi+ \\
& \int_{o_{\phi}\left(\eta_{\mathrm{N}}\right)} \int_{o_{l_{t, e}}\left(\eta_{\mathrm{N}}\right)}\left(-c_{3} l_{t, e}{ }^{2} / H+\left(1-c_{4}\right) l_{t, e}\right) \mathrm{d} l_{t, e} \mathrm{~d} \phi \\
= & \frac{c_{3}}{H} \int_{o_{\phi}\left(\eta_{\mathrm{L}}\right)} \int_{o_{l_{t, e}}\left(\eta_{\mathrm{L}}\right)} l_{t, e}^{2} \mathrm{~d} l_{t, e} \mathrm{~d} \phi+2 c_{4} S_{1}\left(y, \eta_{\mathrm{L}}, r\right)- \\
& \frac{c_{3}}{H} \int_{o_{\phi}\left(\eta_{\mathrm{N}}\right)} \int_{o_{l_{t, e}}\left(\eta_{\mathrm{N}}\right)} l_{t, e}^{2} \mathrm{~d} l_{t, e} \mathrm{~d} \phi+2\left(1-c_{4}\right) S_{1}\left(y, \eta_{\mathrm{N}}, r\right)
\end{aligned}
$$

where $\int_{o_{\phi}(\eta)} \int_{o_{l_{t, e}}(\eta)} l_{t, e}^{2} \mathrm{~d} l_{t, e} \mathrm{~d} \phi+\int_{o^{\prime}{ }_{\phi}(\eta)} \int_{o^{\prime} l_{t, e}(\eta)} l_{t, e}^{2} \mathrm{~d} l_{t, e} \mathrm{~d} \phi=$ $\frac{2}{3} \pi r^{3}$. Given Lemma 1 and dividing (26) by $\pi R_{1}^{2}$, (24) is achieved. Thus, we conclude the proof.

\section{ANALYSIS OF THE SCP}

\section{A. Trend of the SCP with respect to $P_{j}$}

Although the SCP is directly related to $P_{j}$, its trend varies. This can be explained as follows: as $P_{j}$ increases, both $\log _{2}\left(1+\mathrm{SINR}_{s, d}\right)$ and $\log _{2}\left(1+\max _{e \in \Phi}\left(\mathrm{SINR}_{s, e}\right)\right)$ in (7) decrease. If the former decreases faster than the latter, $C_{s}$ decreases; otherwise, $C_{s}$ increases. This will, in turn, affect the performance of the SCP. This is because $C_{s}$ is a variable related to the instantaneous channel state, and the SCP in (8) is the relative average frequency with which the event $C_{s}>\mathcal{R}_{t}$ occurs throughout all instantaneous channel states (assuming the system is ergodic). If $C_{s}$ increases as $P_{j}$ increases for all instantaneous channel states, the SCP increases; if $C_{s}$ decreases as $P_{j}$ increases for all instantaneous channel states, the SCP decreases. Otherwise, the trend of the SCP depends on the trends of $C_{s}$ for the majority of the instantaneous channel states.

\section{B. Trend of the SCP with respect to $\frac{P_{s}}{P_{j}}$}

This subsection investigates the trend of the SCP with respect to transmitting-to-jamming power ratio $\frac{P_{s}}{P_{j}}$ for $d$ and UEDs working in an interference-limited regime. Let variable $x=\frac{P_{s}}{P_{j}}$, and random variables $d_{1}=\frac{l_{s, d}^{-\beta}\left|h_{s, d}\right|^{2}}{l_{j, d}^{-\alpha} \eta_{j, d}}, d_{2}=$ $\max _{e \in \Phi}\left(\frac{l_{s, e}^{-\alpha} \eta_{s, e}}{l_{j, e}^{-\alpha}}\right)$, we have

$$
y=\frac{1+\frac{P_{s, d}}{P_{j, d}}}{1+\max _{e} \in \Phi\left(\frac{P_{s, e}}{P_{j, e}}\right)}=\frac{1+d_{1} x}{1+d_{2} x}
$$

for $x \geq 0$. As shown in Fig. 3, $y$ is a hyperbola for $d_{1}, d_{2}>0$. Its horizontal asymptote is $y=d_{1} / d_{2}$. A special point that the curve passes through the $\mathrm{x}$-axis is $\left(-1 / d_{1}, 0\right)$, and the vertical asymptote of the hyperbola is given by $x=-1 / d_{2}$. Clearly, when $x=0, y=1$ and the achievable secrecy rate is zero.

For $d_{1}>d_{2}$, the hyperbola $y$ is monotonically increasing in $x$, and if $d_{1}<d_{2}, y$ is monotonically decreasing on $x$. Note that (8) defines the statistical comparison between (27) and $2^{\mathcal{R}_{t}}$ where $\mathcal{R}_{t} \geq 0$. For $d_{1}<d_{2}, y$ is always less than one. Hence, it is smaller than $2^{\mathcal{R}_{t}}$. Therefore, when $d_{1}<d_{2}$, neither increasing nor decreasing $P_{s} / P_{j}$ will affect the results of the comparison between (27) and $2^{\mathcal{R}_{t}}$. Consequently, the monotonicity of (8) is solely decided by $d_{1}>d_{2}$. As a result, the SCP is monotonically increasing as $P_{s} / P_{j}$ increases.

Meanwhile, when $\mathcal{R}_{t}=0$, neither increasing nor decreasing $P_{s} / P_{j}$ will affect the results of the comparison between (8) and $2^{\mathcal{R}_{t}}$ because (27) is always larger than one for $d_{1}>d_{2}$, while (27) is always smaller than one for $d_{1}<d_{2}$. To conclude, the analysis above shows that for a given $P_{s}$, the less $P_{j}$ the better the SCP in the interference-limited environment. As we will see in the following section, deploying the UAV jammer to a carefully chosen position would benefit the communication networks than the case without the UAV jammer.

\section{Trend of the SCP with respect to jammer's position}

This subsection investigates the trend of the SCP with respect to jammer's position when all ATG links are in a pure LoS state and $H^{2}>>\left(R_{1}+l_{s, d}\right)^{2}$. An important aspect of UAV networks is the high likelihood of having significantly stronger LoS components than the reflected multipath components in some deployment scenarios (especially in suburban and rural morphologies) [14]. Hence, it is very important to study the performance of the SCP for the ATG links in a pure LoS state. According to (5), for ATG links in a pure LoS state, as $H$ increases, they will remain in a pure LoS state. 


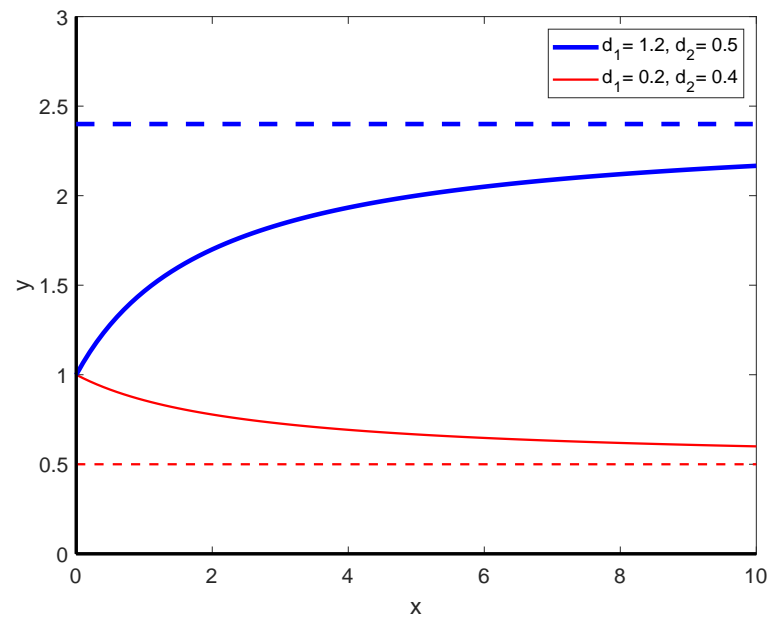

Fig. 3. The plot of hyperbola (27). The solid lines are the hyperbolas in the first quadrant and the dashed lines are horizontal asymptotes.

Given $l_{j, d}^{2}=l_{t, j}^{2}+l_{s, d}^{2}-2 l_{t, j} l_{s, d} \cos \left(\varphi_{j^{\prime}}-\varphi_{d}\right)+H^{2}$ and $l_{s, e}^{2}=H^{2}+l_{t, e}^{2}$, when $H^{2}>>\left(R_{1}+l_{s, d}\right)^{2}$ and $R_{1} \geq l_{t, j}$, we have $l_{j, d} \approx l_{s, e} \approx H$, and

$$
\frac{1+\frac{P_{s, d}}{P_{j, d}+N_{0}}}{1+\max _{e \in \Phi}\left(\frac{P_{s, e}}{P_{j, e}+N_{e}}\right)} \approx \frac{1+\frac{P_{s} l_{s, d}^{-\beta}\left|h_{s, d}\right|^{2}}{P_{j} H^{-\alpha} \eta_{L}+N_{0}(4 \pi / \lambda)^{2}}}{1+\max _{e \in \Phi}\left(\frac{P_{s} \eta_{L} H^{-\alpha}}{P_{j} l_{j, e}^{-\alpha}+N_{e}(4 \pi / \lambda)^{2}}\right)} .
$$

According to (28), the performance of the SCP is related to $\max _{e \in \Phi} l_{j, e}$. For a UED $e$ uniformly distributed within a disk, the PDF of $l_{e, j}$ conditoning on $l_{t, j}$ is proportional to the circular arc of the circle $O\left(j, l_{j, e}\right)$ that is enclosed by the disk $O\left(t, R_{1}\right)$ [31]. Assuming that for a given $l_{t, j}$, the domain of $l_{j, e}$ with nonzero density is $\left[0, Z_{1}\right]$, where $Z_{1}>0$. Then, as $l_{t, j}$ increases by $\delta>0$ and $j$ still stays within the circle $O\left(t, R_{1}\right)$. The new non-zero domain of $l_{j, e}$ is $\left[0, Z_{1}+\delta\right]$, and the density of $l_{j, e}$ is decreasing in $l_{t, j}$ for any $l_{j, e} \in\left[0, \sqrt{R^{2}+l_{t, j}^{2}}\right]$ while it is increasing for any $l_{j, e} \in\left(\sqrt{R^{2}+l_{t, j}^{2}}, Z_{1}\right]$. Thus, the chance to have a higher $l_{j, e}$ increases as $l_{t, j}$ increases. As a result, when the number of the UEDs is one, placing UAV jammer $j$ to $t$ (i.e., $l_{t, j}=0$ ) will provide the optimum SCP for $H^{2}>>\left(R_{1}+l_{s, d}\right)^{2}$.

Meanwhile, for multiple UEDs, we have the following discussion. Let $n$ i.i.d. random variables $X_{1}, \ldots, X_{n}$ denote the distances from $n$ UEDs to $j$, and $Y=\max \left(X_{1}, \ldots, X_{n}\right)$, since the chance for $X_{n}$ to have a higher value increases as $l_{t, j}$ increases for any $n$, the chance for $Y$ to have a higher value increases as $l_{t, j}$ increases. Thus, placing UAV jammer $j$ at $t$ will obtain the optimum SCP for $H^{2}>>\left(R_{1}+l_{s, d}\right)^{2}$.

\section{Simulation Results}

To validate the derivation and the analysis of the SCP with respect to different variables, simulations based on LTE parameters were developed. The parameters used in the simulation are given in Table III unless otherwise specified. The receiver $d$ and UEDs are assumed to have the same noise power. During each Monte Carlo trial, a sample of $n$ locations is obtained from uniformly distributed UEDs. Along with the locations of
TABLE III

SYSTEM PARAMETERS

\begin{tabular}{ll}
\hline Parameter & Value \\
\hline Environment & Suburban \\
Disk radius $R_{1}$ & $500 \mathrm{~m}$ \\
Receiver location $d$ & $(100,0,0)$ \\
Coding gain & $0 \mathrm{~dB}$ \\
Tx/Rx antenna gain & $0 \mathrm{dBi}$ \\
Receiver noise figure & $9 \mathrm{~dB}$ \\
Carrier frequency & $2.0 \mathrm{GHz}$ \\
Spectrum allocation & $20 \mathrm{MHz}$ \\
Duplex mode & $\mathrm{Half} \mathrm{duplex}$ \\
Thermal noise power density & $-174 \mathrm{dBm} / \mathrm{Hz}$ \\
Path loss exponent $\beta$ & 3 \\
Transmit power $P_{s}$ & $0.1 \mathrm{~W}$ \\
Target secrecy rate $\mathcal{R}_{t}$ & $1 \mathrm{bit} / \mathrm{s} / \mathrm{Hz}$ \\
Number of UEDs $n$ & 2 \\
\hline
\end{tabular}

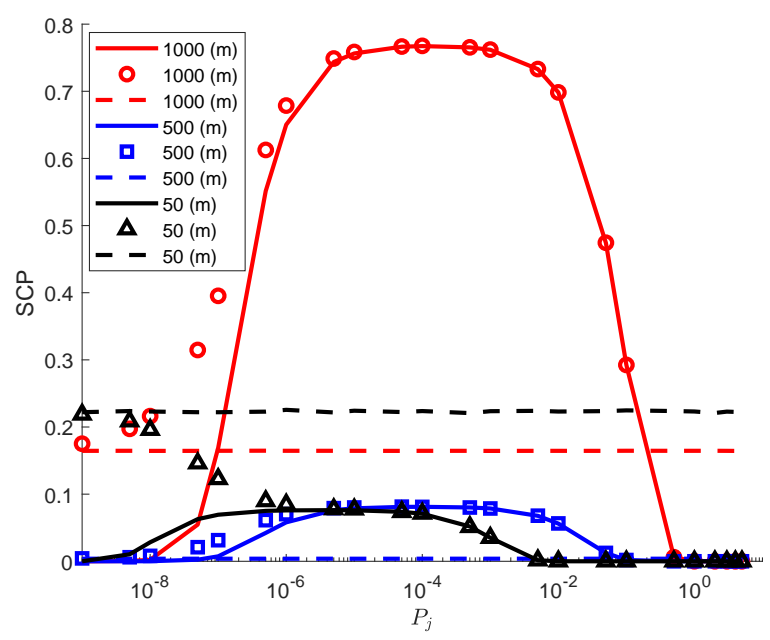

Fig. 4. SCP vs. $P_{j}$ in watts for $H$ given by the figure legends. For the same color, the solid line represents the numerical results with the UAV jammer at $(0,0, H)$, the markers represent the corresponding simulation results, and the dashed line represents the simulated benchmark case of no jammer.

the UAV jammer and receiver, $1 \times 10^{4}$ different instantaneous channel states are generated, which characterize the random channel statistics due to Rayleigh fading and the probabilistic LoS and NLoS channel conditions. Then, the corresponding $C_{s}$ based on each instantaneous channel state is achieved, and the average results on the event $C_{s}>\mathcal{R}_{t}$ throughout all generated instantaneous channel states are saved in one trial. The simulation results are obtained by averaging over $1 \times 10^{5}$ independent Monte Carlo trials.

Fig. 4 shows the performance of the SCP in the simulation for UEDs at different heights. The numerical results in this section are obtained by evaluating (17) for the same parameters as the simulations. One can easily see that the numerical results act as the lower bound to the simulation results of the SCP when $P_{j}$ is small. The simulation curves match well with the numerical results as $P_{j}$ reaches the interference-limited regime. In this regime, the performance of the SCP is decreasing as $P_{j}$ increases. This can be easily explained by the discussion on $P_{s} / P_{j}$ in (27). Meanwhile, the same figure also shows that introducing a UAV jammer to the ground communications could change the performance of the SCP significantly. On the one hand, for $H=500 \mathrm{~m}$ and $1000 \mathrm{~m}$, the SCP increases as $P_{j}$ increases until around $10^{-3} \mathrm{~W}$ and then starts to decrease until the SCP reaches zero. Compared to the benchmark case of no jammer, introducing a UAV jammer with proper $P_{j}$ would allow 


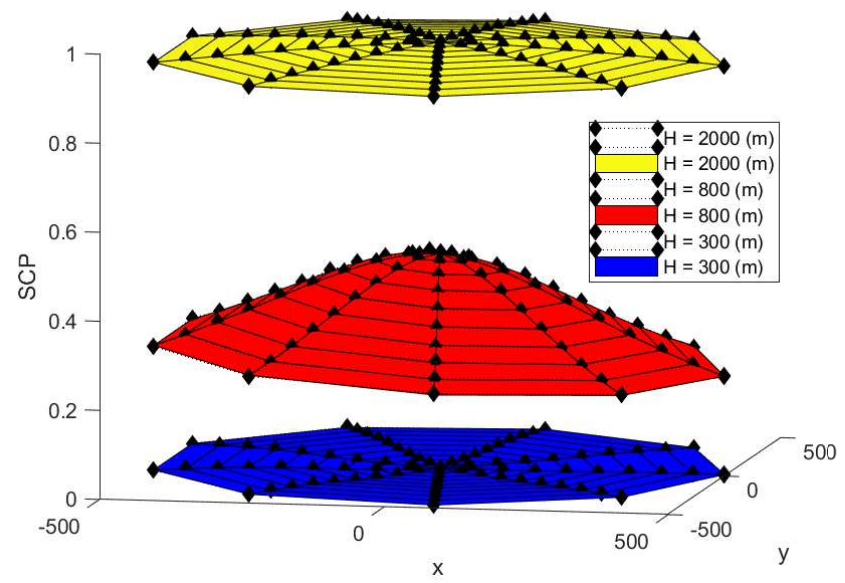

Fig. 5. SCP vs. UAV jammer at $(x, y, H) . P_{j}=0.01 \mathrm{~W}$. The markers represent the simulation results, and the mesh grids represent the numerical results.

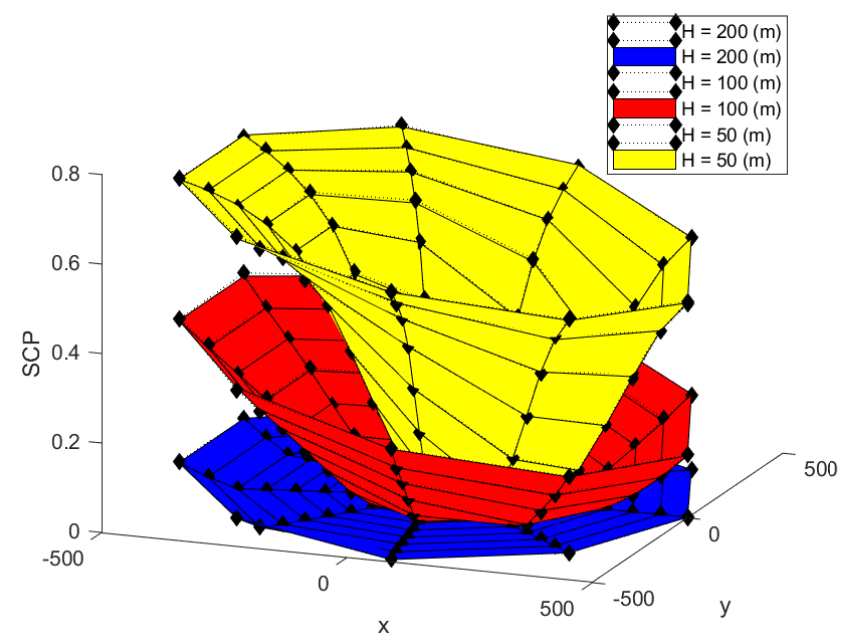

Fig. 6. SCP vs. UAV jammer at $(x, y, H) . P_{j}=0.01 \mathrm{~W}$. The markers represent the simulation results, and the mesh grids represent the numerical results.

the ground communications to achieve a higher SCP. For $H=$ $500 \mathrm{~m}$ and $1000 \mathrm{~m}$, the ground communications with a UAV jammer allow the maximum of $8 \%$ and $60 \%$ increase in SCP, respectively, over the case without a UAV jammer as given by the dashed lines. On the other hand, for $H=50 \mathrm{~m}$, the SCP decreases as $P_{j}$ increases. By having a UAV jammer in this height, the SCP of the ground communications will be affected severely when the UAV jammer is enabled. Meanwhile, noticing the case without the UAV jammer, the achieved SCP in dashed lines show that the SCP at $H=50 \mathrm{~m}$ is higher than the one at $H=500 \mathrm{~m}$ and $1000 \mathrm{~m}$. It is because increasing $H$ will cause not only a higher LoS probability from the transmitter to a UED but also a longer receiving distance (i.e., a higher path loss). In general, the ground communications prefer to have NLoS links towards the UEDs and have a maximum distance from it, however, these two objectives conflicts with each other. If the LoS probability from the transmitter to a UED contributes more than the path loss does to the SCP as $H$ increases, the SCP will decrease. Otherwise, the SCP will increase.

Fig. 5 depicts the simulation results on the SCP when all

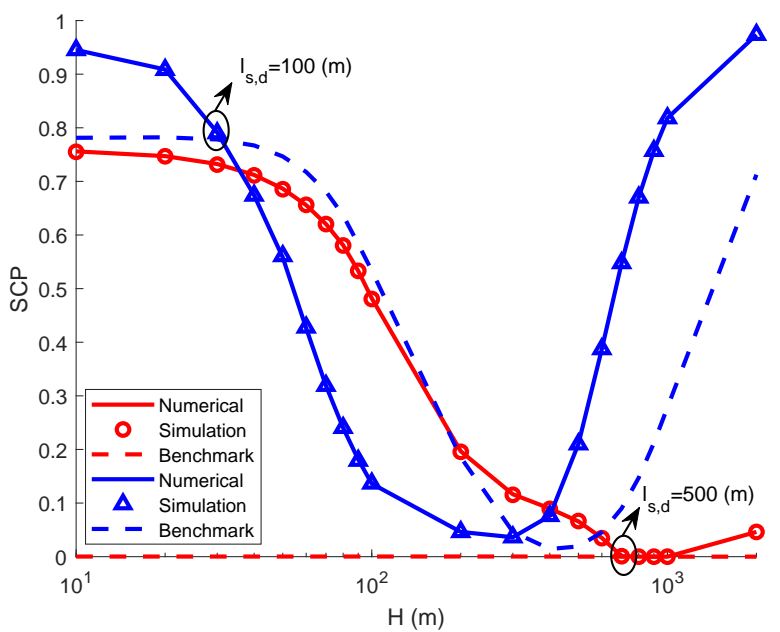

Fig. 7. SCP vs. $H$ for different $l_{s, d} . P_{j}=0.01 \mathrm{~W}$, and the UAV jammer is located at $(0,0, H)$.

ATG links are in a pure LoS state. When $H=2000 \mathrm{~m}$, the performance of the SCP is optimum when placing the UAV jammer at $(0,0, H)$. This can be explained by the discussion on (28). The performance curves from $H=300$ to $H=2000$ show that, for the UAV jammer with the same $\mathrm{x}$ and $\mathrm{y}$ coordinates, the SCP increases as $H$ increases. This can also be explained by (28), where the right term decreases as $H$ increases for the ATG links in a pure LoS state. Meanwhile, Fig. 6 gives the cases without the requirement on a pure LoS state for ATG links. In general, the trend of the SCP with respect to the height of UAVs is hard to capture, because increasing $H$ will not only cause a higher LoS probability but also decreases the jamming and received signal powers. A higher LoS probability from $s$ to UED will cause the SCP to drop while a smaller jamming power will cause the SCP to increase. Meanwhile, the surface shows that the jammer should move away from the receiver $d$ in order to achieve a higher SCP. Furthermore, by observing both Fig. 5 and Fig. 6, the numerical results of the SCP matching with the simulation results suggest that both receiver $d$ and UEDs are working in the interference-limited regime for $P_{j}=0.01 \mathrm{~W}$. By increasing $H$ from $50 \mathrm{~m}$ to $2000 \mathrm{~m}$, the SCP at the given $\mathrm{x}$ and $\mathrm{y}$ coordinates decreases first and then increases.

As shown in Fig. 7, the UAV jammer will affect the SCP heavily as compared to the benchmark case of no jammer for different $l_{s, d}$. For a ground link distance $l_{s, d}=100 \mathrm{~m}$, the SCP changes significantly for different $H$ in the benchmark. This is because a larger $H$ causes not only the larger average path loss, but also the higher LoS probability from the transmitter $s$ to the UEDs. Because the benchmarked SCP for $l_{s, d}=100$ $\mathrm{m}$ is decreasing as $H$ increases until around $400 \mathrm{~m}$ in Fig. 7, the effect of the LoS probability on the SCP is stronger than that of the path loss from the transmitter $s$ to the UEDs. This trend reverses after $H=400 \mathrm{~m}$ due to the stronger effect of the path loss than that of the LoS probability. Then, both the simulation and numerical results reveal that, when $l_{s, d}=100 \mathrm{~m}$, the ground link can increase its SCP to at most $15 \%$ for $H=10$ $\mathrm{m}$ to $30 \mathrm{~m}$ and for $H=300 \mathrm{~m}$ to $2000 \mathrm{~m}$ in the presence of UAV jammer. Meanwhile, between $30 \mathrm{~m}$ and $300 \mathrm{~m}$, the SCP decreases when comparing with the benchmark. This implies 
that the UAV jammer has a stronger influence to the receiver than to the UEDs in this range. Meanwhile, for a ground link distance $l_{s, d}=500 \mathrm{~m}$, where the corresponding path loss from the transmitter $s$ to the receiver $d$ is very large, the SCP is close to zero for the benchmark and the ground link is more susceptible to eavesdropping when compared to $l_{s, d}=100 \mathrm{~m}$. Then, by enabling the UAV jammer, the a maximum of $75 \%$ increase can be achieved in the SCP for the given setting.

\section{CONCLUSION}

In this paper, we investigated the secrecy performance of the ground link in the presence of randomly deployed noncolluding UEDs. A piecewise fitting model has been proposed to characterize the LoS probability of an ATG link with higher accuracy than the existing sigmoid fitting. The SCP has been formulated based on the piecewise fitting model so that the formulation become tractable. The performance of the SCP with respect to transmitting-to-jamming power ratio, the height of the UAVs and the UAV jammer location has been investigated. The effective jamming power and location of the UAV jammer have been pointed out. A combination of the optimum jamming position and power is expected to achieve the optimum secrecy performance, and we leave the optimization problem to find the best jamming position and/or power for both the LoS and NLoS link states to our future work. The model and analysis frameworks presented in this paper will help to facilitate the analysis of the UAV-aided communications.

\section{APPENDIX}

Lemma 1. For a UED $e$ which is uniformly distributed within the disk $O(t, r)$, denote $\phi=\varphi_{j^{\prime}}-\varphi_{e^{\prime}}$ and $\eta$ as the excess path loss, the domains of $\phi$ and $l_{t, e}$ so that $1+\frac{\left(H^{2}+l_{t, e}^{2}\right)^{\frac{-\alpha}{2}} \eta P_{s}}{\left(l_{t, j}^{2}+l_{t, e}^{2}-2 l_{t, j} l_{t, e} \cos \phi\right)^{\frac{-\alpha}{2}} P_{j}} \leq y$ are denoted as $\left\{o_{\phi}(\eta), o_{l_{t, e}}(\eta)\right\}$, while the domains of $\phi$ and $l_{t, e}$ so that $1+\frac{\left(H^{2}+l_{t, e}^{2}\right)^{\frac{-\alpha}{2}} \eta P_{s}}{\left(l_{t, j}^{2}+l_{t, e}^{2}-2 l_{t, j} l_{t, e} \cos \phi\right)^{\frac{-\alpha}{2}} P_{j}}>y$ are denoted as $\left\{o_{\phi}^{\prime}(\eta), o^{\prime}{ }_{t, e}(\eta)\right\}$. Let $\mathcal{F}_{1}=\cos ^{-1} \frac{-\sqrt{B A}}{l_{t, j}}, \mathcal{F}_{2}=\cos ^{-1} \frac{r^{2} A+B}{2 r l_{t, j}}$, $A=1-\left((y-1) \frac{P_{j}}{\eta P_{s}}\right)^{\frac{2}{\alpha}}, B=l_{t, j}^{2}-(1-A) H^{2} \cdot x_{1}=$ $\frac{l_{t, j} \cos \phi+\sqrt{\left(l_{t, j} \cos \phi\right)^{2}-B A}}{A}$, and $x_{2}=\frac{l_{t, j} \cos \phi-\sqrt{\left(l_{t, j} \cos \phi\right)^{2}-B A}}{A}$.

Based on different $A$ and $B$, these domains are given as follows.

For $A<0, B \leq 0$, we have $o^{\prime} l_{t, e}(\eta)=\left[x_{1}, x_{2}\right]$ and

$$
o_{\phi}^{\prime}(\eta)= \begin{cases}{\left[\mathcal{F}_{1}, \mathcal{F}_{2}\right],} & \text { if } r^{2} A \leq B, \frac{-r^{2} A-B}{2 r} \leq l_{t, j} \\ {\left[\mathcal{F}_{1}, \pi\right],} & \text { if } r^{2} A<B, \sqrt{B A} \leq l_{t, j}<\frac{-r^{2} A-B}{2 r} \\ \{\emptyset\}, & \text { otherwise }\end{cases}
$$

or $o^{\prime}{ }_{t, e}(\eta)=\left[x_{1}, r\right]$ and

$$
o_{\phi}^{\prime}(\eta)= \begin{cases}\left(\mathcal{F}_{2}, \pi\right], & \text { if } r^{2} A \leq B, \frac{-r^{2} A-B}{2 r} \leq l_{t, j} \\ {\left[\mathcal{F}_{2}, \pi\right],} & \text { if } r^{2} A>B, \frac{-r^{2} A-B}{2 r} \leq l_{t, j} \\ \{\emptyset\}, & \text { otherwise. }\end{cases}
$$

For $A>0, B \geq 0$, we have $o_{l_{t, e}}(\eta)=\left[x_{2}, x_{1}\right]$ and

$o_{\phi}(\eta)= \begin{cases}{\left[\mathcal{F}_{2}, \pi-\mathcal{F}_{1}\right],} & \text { if } r^{2} A \geq B, l_{t, j} \geq \frac{r^{2} A+B}{2 r} \\ {\left[0, \pi-\mathcal{F}_{1}\right],} & \text { if } r^{2} A>B, \sqrt{B A} \leq l_{t, j}<\frac{r^{2} A+B}{2 r} \\ \{\emptyset\}, & \text { otherwise }\end{cases}$

or $o_{l_{t, e}}(\eta)=\left[x_{2}, r\right]$ and

$$
o_{\phi}(\eta)= \begin{cases}{\left[0, \mathcal{F}_{2}\right],} & \text { if } r^{2} A \leq B, \frac{r^{2} A+B}{2 r} \leq l_{t, j} \\ {\left[0, \mathcal{F}_{2}\right),} & \text { if } r^{2} A>B, \frac{r^{2} A+B}{2 r} \leq l_{t, j} \\ \{\emptyset\}, & \text { otherwise. }\end{cases}
$$

For $A<0, B>0$, we have $o^{\prime} l_{t, e}(\eta)=[0, r]$ and

$$
o_{\phi}^{\prime}(\eta)= \begin{cases}{[0, \pi],} & \text { if }-r^{2} A<B, l_{t, j}<\frac{r^{2} A+B}{2 r} \\ \left(\mathcal{F}_{2}, \pi\right], & \text { if } l_{t, j} \geq \frac{\left|r^{2} A+B\right|}{2 r} \\ \{\emptyset\}, & \text { otherwise; }\end{cases}
$$

or $o^{\prime}{ }_{t, e}(\eta)=\left[0, x_{2}\right]$ and

$$
o_{\phi}^{\prime}(\eta)= \begin{cases}{[0, \pi],} & \text { if }-r^{2} A>B, l_{t, j}<\frac{-r^{2} A-B}{2 r} \\ {\left[0, \mathcal{F}_{2}\right],} & \text { if } l_{t, j} \geq \frac{\left|r^{2} A+B\right|}{2 r} \\ \{\emptyset\}, & \text { otherwise. }\end{cases}
$$

For $A>0, B<0$, we have $o_{l_{t, e}}(\eta)=[0, r]$ and

$$
o_{\phi}(\eta)= \begin{cases}{[0, \pi],} & \text { if }-r^{2} A>B, l_{t, j}<\frac{-r^{2} A-B}{2 r} \\ {\left[0, \mathcal{F}_{2}\right],} & \text { if } l_{t, j} \geq \frac{\left|r^{2} A+B\right|}{2 r} \\ \{\emptyset\}, & \text { otherwise; }\end{cases}
$$

or $o_{l_{t, e}}(\eta)=\left[0, x_{1}\right]$ and

$$
o_{\phi}(\eta)= \begin{cases}{[0, \pi],} & \text { if }-r^{2} A<B, l_{t, j}<\frac{r^{2} A+B}{2 r} \\ {\left[\mathcal{F}_{2}, \pi\right],} & \text { if } l_{t, j} \geq \frac{\left|r^{2} A+B\right|}{2 r} \\ \{\emptyset\}, & \text { otherwise. }\end{cases}
$$

For $A=0$, we have $o_{l_{t, e}}(\eta)=\left[\frac{\left|l_{t, j}^{2}-H^{2}\right|}{2 l_{t, j}}, r\right]$ and

$$
o_{\phi}(\eta)= \begin{cases}{\left[0, \cos ^{-1}\left(\frac{l_{t, j}^{2}-H^{2}}{2 l_{t, j} l_{t, e}}\right)\right],} & \text { if } \frac{\left|l_{t, j}^{2}-H^{2}\right|}{2 l_{t, j}} \leq r \\ \{\emptyset\}, & \text { otherwise; }\end{cases}
$$

or $o_{l_{t, e}}(\eta)=\left[0, \frac{-l_{t, j}^{2}+H^{2}}{2 l_{t, j}}\right)$ and

$$
o_{\phi}(\eta)= \begin{cases}{[0, \pi],} & \text { if } r \geq \frac{-l_{t, j}^{2}+H^{2}}{2 l_{t, j}} \\ \{\emptyset\}, & \text { otherwise; }\end{cases}
$$

or $o_{l_{t, e}}(\eta)=[0, r]$ and

$$
o_{\phi}(\eta)= \begin{cases}{[0, \pi],} & \text { if } r<\frac{-l_{t, j}^{2}+H^{2}}{2 l_{t, j}} \\ \{\emptyset\}, & \text { otherwise. }\end{cases}
$$

Proof: For $A \neq 0$, the corresponding solutions of $l_{t, e}$ in $1+\frac{\left(H^{2}+l_{t, e}^{2}\right)^{\frac{-\alpha}{2}} \eta P_{s}}{\left(l_{t, j}^{2}+l_{t, e}^{2}-2 l_{t, j} l_{t, e} \cos \phi\right)^{\frac{-\alpha}{2}} P_{j}}=y$ are denoted as

$$
\begin{aligned}
& x_{1}=\frac{l_{t, j} \cos \phi+\sqrt{\left(l_{t, j} \cos \phi\right)^{2}-B A}}{A} \\
& x_{2}=\frac{l_{t, j} \cos \phi-\sqrt{\left(l_{t, j} \cos \phi\right)^{2}-B A}}{A}
\end{aligned}
$$


where $x_{1} \leq x_{2}$ for $A<0$, while $x_{2} \leq x_{1}$ for $A>0$. For $x_{1}$ and $x_{2}$ to exist,

$$
\left(l_{t, j} \cos \phi\right)^{2}-B A \geq 0 .
$$

As a result, $\cos \phi \geq \frac{\sqrt{B A}}{l_{t, j}}$ or $\cos \phi \leq-\frac{\sqrt{B A}}{l_{t, j}}$ for $B A \geq 0$, and $\left(l_{t, j} \cos \phi\right)^{2} \geq 0$ for $B A<0$. Since $\phi=\varphi_{j^{\prime}}-\varphi_{e^{\prime}}$ and the impact from $j$ to $e$ for $\phi \in[0, \pi]$ is the same as the case for $\phi \in[\pi, 2 \pi]$. Thus, we only consider $\phi \in[0, \pi]$ in the following analysis. The domains of $\phi$ in (41) are given by

$$
\begin{gathered}
\phi \leq \pi-\mathcal{F}_{1}, \text { for } l_{t, j} \geq \sqrt{B A}, B A \geq 0, \\
\phi \geq \mathcal{F}_{1}, \text { for } l_{t, j} \geq \sqrt{B A}, B A \geq 0, \\
\phi \in[0, \pi], \text { for } B A<0 .
\end{gathered}
$$

Meanwhile, $1+\frac{\left(H^{2}+l_{t, e}^{2}\right)^{\frac{-\alpha}{2}} \eta P_{s}}{\left(l_{t, j}^{2}+l_{t, e}^{2}-2 l_{t, j} l_{t, e} \cos \phi\right)^{\frac{-\alpha}{2}} P_{j}} \leq y$ gives

$$
A l_{t, e}^{2}-2 l_{t, j} \cos (\phi) l_{t, e}+l_{t, j}^{2}-(1-A) H^{2} \leq 0 .
$$

Apparently, when $A<0, l_{t, e} \geq x_{2}$ or $l_{t, e} \leq x_{1}$; when $A>0, x_{2} \leq l_{t, e} \leq x_{1}$. To simplify analysis, we investigate $x_{1} \leq l_{t, e} \leq x_{2}$ for $A<0$ and $x_{2} \leq l_{t, e} \leq x_{1}$ for $A>0$. As a result, the former is the case for $A<0$ and

$$
A l_{t, e}^{2}-2 l_{t, j} \cos (\phi) l_{t, e}+l_{t, j}^{2}-(1-A) H^{2} \geq 0 .
$$

For $A<0, B \leq 0$ : Assuming that (41) holds, comparing $x_{1}$ with 0 gives

$$
\phi \in\left[\frac{\pi}{2}, \pi\right], \text { for } x_{1} \geq 0 ; \text { and } \phi \in\left[0, \frac{\pi}{2}\right] \text {, for } x_{1}<0 \text {. }
$$

Assuming that (41) holds, comparing $x_{2}$ with 0 and considering that $x_{1} \leq x_{2}$ for $A<0$, we have

$$
\phi \in\left[\frac{\pi}{2}, \pi\right], x_{2} \geq 0 .
$$

Comparing $x_{1}$ with $r$, we have

$$
x_{1} \leq r \Rightarrow \sqrt{\left(l_{t, j} \cos \phi\right)^{2}-B A} \geq r A-l_{t, j} \cos \phi .
$$

If

$$
r A-l_{t, j} \cos \phi \geq 0
$$

then

$$
\phi \in \begin{cases}{\left[\mathcal{F}_{3}, \pi\right],} & \text { if } l_{t, j} \geq-r A \\ \{\emptyset\}, & \text { if } l_{t, j}<-r A\end{cases}
$$

where $\mathcal{F}_{3}=\cos ^{-1} \frac{r A}{l_{t, j}}$.

Assuming that (41) and (50) hold, solving (49) gives

$$
\phi \in \begin{cases}{\left[\mathcal{F}_{2}, \pi\right],} & \text { if } l_{t, j} \geq \frac{-r^{2} A-B}{2 r} \\ \{\emptyset\}, & \text { otherwise. }\end{cases}
$$

Combining (51) and (52) with intersections yields

$$
\phi \in \begin{cases}{\left[\mathcal{F}_{3}, \pi\right],} & \text { if } r^{2} A \leq B, l_{t, j} \geq-r A \\ {\left[\mathcal{F}_{2}, \pi\right],} & \text { if } r^{2} A>B, l_{t, j} \geq \frac{-r^{2} A-B}{2 r} \\ \{\emptyset\}, & \text { otherwise. }\end{cases}
$$

Combining (53) and (43) with intersections yields

$$
\phi \in \begin{cases}{\left[\mathcal{F}_{3}, \pi\right],} & \text { if } r^{2} A \leq B, l_{t, j} \geq-r A \\ {\left[\mathcal{F}_{2}, \pi\right],} & \text { if } r^{2} A>B, l_{t, j} \geq \frac{-r^{2} A-B}{2 r} \\ \{\emptyset\}, & \text { otherwise. }\end{cases}
$$

If $r A-l_{t, j} \cos \phi<0$, then

$$
\phi \in \begin{cases}{[0, \pi],} & \text { if } l_{t, j}<-r A \\ {\left[0, \mathcal{F}_{3}\right),} & \text { if } l_{t, j} \geq-r A .\end{cases}
$$

Combining (55) and (43) with intersections yields

$$
\phi \in \begin{cases}{\left[\mathcal{F}_{1}, \pi\right],} & \text { if } r^{2} A<B, \sqrt{B A} \leq l_{t, j}<-r A \\ {\left[\mathcal{F}_{1}, \mathcal{F}_{3}\right),} & \text { if } r^{2} A<B, l_{t, j} \geq-r A \\ \{\emptyset\}, & \text { otherwise. }\end{cases}
$$

The unions of (54) and (56) give

$$
\phi \in \begin{cases}{\left[\mathcal{F}_{2}, \pi\right],} & \text { if } r^{2} A>B, l_{t, j} \geq \frac{-r^{2} A-B}{2 r} \\ {\left[\mathcal{F}_{1}, \pi\right],} & \text { if } r^{2} A \leq B, \sqrt{B A} \leq l_{t, j} \\ \{\emptyset\}, & \text { otherwise. }\end{cases}
$$

Comparing $r$ with $x_{2}$,

$$
x_{2}>r \Rightarrow \sqrt{\left(l_{t, j} \cos \phi\right)^{2}-B A}>-r A+l_{t, j} \cos \phi .
$$

If

$$
-r A+l_{t, j} \cos \phi \geq 0
$$

then

$$
\phi \in \begin{cases}{[0, \pi],} & \text { if } l_{t, j}<-r A \\ {\left[0, \mathcal{F}_{3}\right],} & \text { if } l_{t, j} \geq-r A .\end{cases}
$$

Assuming that (41) and (59) hold, solving (58) gives

$$
\phi \in \begin{cases}\left(\mathcal{F}_{2}, \pi\right], & \text { if } l_{t, j} \geq \frac{-r^{2} A-B}{2 r} \\ \{\emptyset\}, & \text { otherwise. }\end{cases}
$$

Combining (60) and (61) with intersections yields

$\phi \in \begin{cases}\left(\mathcal{F}_{2}, \pi\right], & \text { if } r^{2} A<B, \frac{-r^{2} A-B}{2 r} \leq l_{t, j}<-r A \\ \left(\mathcal{F}_{2}, \mathcal{F}_{3}\right], & \text { if } r^{2} A<B, l_{t, j} \geq-r A \\ \{\emptyset\}, & \text { otherwise. }\end{cases}$

Then, the intersections of (62) and (43) yield (62).

If $-r A+l_{t, j} \cos \phi<0$, then

$$
\phi \in \begin{cases}\left(\mathcal{F}_{3}, \pi\right], & \text { if } l_{t, j} \geq-r A \\ \{\emptyset\}, & \text { otherwise. }\end{cases}
$$

Then, combining (63) and (43) with intersections yields

$$
\phi \in \begin{cases}\left(\mathcal{F}_{3}, \pi\right], & \text { if } r^{2} A \leq B, l_{t, j} \geq-r A \\ {\left[\mathcal{F}_{1}, \pi\right],} & \text { if } r^{2} A>B, l_{t, j} \geq \sqrt{B A} \\ \{\emptyset\}, & \text { otherwise. }\end{cases}
$$

Therefore, the regions for $x_{2}>r$ are given by the unions of (62) and (64):

$$
\phi \in \begin{cases}\left(\mathcal{F}_{2}, \pi\right], & \text { if } r^{2} A \leq B, \frac{-r^{2} A-B}{2 r} \leq l_{t, j} \\ {\left[\mathcal{F}_{1}, \pi\right],} & \text { if } r^{2} A>B, l_{t, j} \geq \sqrt{B A} \\ \{\emptyset\}, & \text { otherwise. }\end{cases}
$$

Meanwhile,

$$
\begin{aligned}
& \quad x_{2} \leq r \Rightarrow \sqrt{\left(l_{t, j} \cos \phi\right)^{2}-B A} \leq-r A+l_{t, j} \cos \phi . \\
& \text { If }-r A+l_{t, j} \cos \phi \geq 0 \text {, we have (60). }
\end{aligned}
$$


Assuming that (41) and (60) hold, solving (66) gives

$$
\phi \in \begin{cases}{\left[0, \mathcal{F}_{2}\right],} & \text { if } l_{t, j} \geq \frac{-r^{2} A-B}{2 r} \\ {[0, \pi],} & \text { if } l_{t, j}<\frac{-r^{2} A-B}{2 r} .\end{cases}
$$

Combining (60) and (67) with intersections yields

$$
\phi \in \begin{cases}{\left[0, \mathcal{F}_{2}\right],} & \text { if } r^{2} A \leq B, l_{t, j} \geq \frac{-r^{2} A-B}{2 r} \\ {\left[0, \mathcal{F}_{3}\right],} & \text { if } r^{2} A>B, l_{t, j} \geq-r A \\ {[0, \pi],} & \text { if } r^{2} A \leq B, l_{t, j}<\frac{-r^{2} A-B}{2 r} \\ {[0, \pi],} & \text { if } r^{2} A>B, l_{t, j}<-r A \\ \{\emptyset\}, & \text { otherwise. }\end{cases}
$$

The intersections of (68) and (43) are given by (29). As a result, when $0 \leq x_{1} \leq r$ and $x_{2} \leq r$, we have (29). Meanwhile, when $0 \leq x_{1} \leq r$ and $x_{2}>r$, we have (30).

For $A>0, B \geq 0$ : Assuming that (41) holds, comparing $x_{2}$ with 0 gives

$\phi \in\left[\frac{\pi}{2}, \pi\right]$, for $x_{2}<0 ;$ and $\phi \in\left[0, \frac{\pi}{2}\right]$, for $x_{2} \geq 0$.

Assuming that (41) holds, comparing $x_{1}$ with 0 gives $\phi \in\left[\frac{\pi}{2}, \pi\right]$, for $x_{1}<0 ;$ and $\phi \in\left[0, \frac{\pi}{2}\right]$, for $x_{1} \geq 0$.

Since $x_{1}$ must be larger than 0 for a valid region to exist, $\phi \in\left[0, \frac{\pi}{2}\right]$ and (42) stands.

Comparing $x_{2}$ with $r$, we have

$$
x_{2} \leq r \Rightarrow \sqrt{\left(l_{t, j} \cos \phi\right)^{2}-B A} \geq l_{t, j} \cos \phi-r A
$$

If

$$
l_{t, j} \cos \phi-r A \geq 0,
$$

then

$$
\phi \in \begin{cases}{\left[0, \mathcal{F}_{3}\right],} & \text { if } l_{t, j} \geq r A \\ \{\emptyset\}, & \text { if } l_{t, j}<r A .\end{cases}
$$

Assuming that (41) and (72) hold, solving (71) gives

$$
\phi \in \begin{cases}{\left[0, \mathcal{F}_{2}\right],} & \text { if } l_{t, j} \geq \frac{r^{2} A+B}{2 r} \\ \{\emptyset\}, & \text { if } l_{t, j}<\frac{r^{2} A+B}{2 r} .\end{cases}
$$

Then, combining (73), (74) and (42) with intersections yields

$$
\phi \in \begin{cases}{\left[0, \mathcal{F}_{3}\right],} & \text { if } r^{2} A>B, l_{t, j} \geq r A \\ {\left[0, \mathcal{F}_{2}\right],} & \text { if } r^{2} A \leq B, l_{t, j} \geq \frac{r^{2} A+B}{2 r} \\ \{\emptyset\}, & \text { otherwise. }\end{cases}
$$

If $l_{t, j} \cos \phi-r A<0$, then

$$
\phi \in \begin{cases}{[0, \pi],} & \text { if } l_{t, j}<r A \\ \left(\mathcal{F}_{3}, \pi\right], & \text { if } l_{t, j} \geq r A .\end{cases}
$$

Then, combining (76) and (42) with intersections yields

$$
\phi \in \begin{cases}{\left[0, \pi-\mathcal{F}_{1}\right],} & \text { if } r^{2} A>B, \sqrt{B A} \leq l_{t, j}<r A \\ \left(\mathcal{F}_{3}, \pi-\mathcal{F}_{1}\right], & \text { if } r^{2} A>B, r A \leq l_{t, j} \\ \{\emptyset\}, & \text { otherwise. }\end{cases}
$$

The unions of (75) and (77) yield

$$
\phi \in \begin{cases}{\left[0, \mathcal{F}_{2}\right],} & \text { if } r^{2} A \leq B, l_{t, j} \geq \frac{r^{2} A+B}{2 r} \\ {\left[0, \pi-\mathcal{F}_{1}\right],} & \text { if } r^{2} A>B, \sqrt{B A} \leq l_{t, j} \\ \{\emptyset\}, & \text { otherwise. }\end{cases}
$$

Comparing $r$ with $x_{1}$,

$$
x_{1} \leq r \Rightarrow \sqrt{\left(l_{t, j} \cos \phi\right)^{2}-B A} \leq r A-l_{t, j} \cos \phi .
$$

If

$$
r A-l_{t, j} \cos \phi \geq 0
$$

then

$$
\phi \in \begin{cases}{[0, \pi],} & \text { if } l_{t, j}<r A \\ {\left[\mathcal{F}_{3}, \pi\right],} & \text { if } l_{t, j} \geq r A .\end{cases}
$$

Assuming that (41) and (80) hold, solving (79) gives

$$
\phi \in \begin{cases}{[0, \pi],} & \text { if } l_{t, j}<\frac{r^{2} A+B}{2 r} \\ {\left[\mathcal{F}_{2}, \pi\right],} & \text { if } l_{t, j} \geq \frac{r^{2} A+B}{2 r} .\end{cases}
$$

Combining (82) and (81) with intersections yields

$$
\phi \in \begin{cases}{[0, \pi],} & \text { if } r^{2} A<B, l_{t, j}<r A \\ {[0, \pi],} & \text { if } r^{2} A \geq B, l_{t, j}<\frac{r^{2} A+B}{2 r} \\ {\left[\mathcal{F}_{3}, \pi\right],} & \text { if } r^{2} A<B, r A \leq l_{t, j} \\ {\left[\mathcal{F}_{2}, \pi\right],} & \text { if } r^{2} A \geq B, \frac{r^{2} A+B}{2 r} \leq l_{t, j} \\ \{\emptyset\}, & \text { otherwise. }\end{cases}
$$

Then, combining (83) and (42) with intersections yields

$$
\begin{aligned}
& \phi \in \begin{cases}{\left[\mathcal{F}_{2}, \pi-\mathcal{F}_{1}\right],} & \text { if } r^{2} A \geq B, l_{t, j} \geq \frac{r^{2} A+B}{2 r} \\
{\left[0, \pi-\mathcal{F}_{1}\right],} & \text { if } r^{2} A \geq B, \sqrt{B A} \leq l_{t, j}<\frac{r^{2} A+B}{2 r} \\
\{\emptyset\}, & \text { otherwise. }\end{cases} \\
& x_{1}>r \Rightarrow \sqrt{\left(l_{t, j} \cos \phi\right)^{2}-B A}>r A-l_{t, j} \cos \phi .
\end{aligned}
$$

If

$$
r A-l_{t, j} \cos \phi \geq 0
$$

we have (81).

Assuming that (41) and (86) hold, solving (85) gives

$$
\phi \in \begin{cases}{\left[0, \mathcal{F}_{2}\right),} & \text { if } l_{t, j} \geq \frac{r^{2} A+B}{2 r} \\ \{\emptyset\}, & \text { otherwise. }\end{cases}
$$

Combining (81) and (87) with intersections yields

$$
\phi \in \begin{cases}{\left[\mathcal{F}_{3}, \mathcal{F}_{2}\right),} & \text { if } r^{2} A>B, l_{t, j} \geq r A \\ {\left[0, \mathcal{F}_{2}\right),} & \text { if } r^{2} A>B, \frac{r^{2} A+B}{2 r} \leq l_{t, j}<r A \\ \{\emptyset\}, & \text { otherwise. }\end{cases}
$$

If $r A-l_{t, j} \cos \phi<0$, we have

$$
\phi \in \begin{cases}{\left[0, \mathcal{F}_{3}\right),} & \text { if } l_{t, j} \geq r A \\ \{\emptyset\}, & \text { otherwise. }\end{cases}
$$

The unions of (88) and (89) yield

$$
\phi \in \begin{cases}{\left[0, \mathcal{F}_{2}\right),} & \text { if } r^{2} A>B, l_{t, j} \geq \frac{r^{2} A+B}{2 r} \\ {\left[0, \mathcal{F}_{3}\right),} & \text { if } r^{2} A \leq B, l_{t, j} \geq r A \\ \{\emptyset\}, & \text { otherwise. }\end{cases}
$$

Then, combining (90) and (42) with intersections yields

$$
\phi \in \begin{cases}{\left[0, \mathcal{F}_{2}\right),} & \text { if } r^{2} A>B, l_{t, j} \geq \frac{r^{2} A+B}{2 r} \\ {\left[0, \pi-\mathcal{F}_{1}\right],} & \text { if } r^{2} A \leq B, l_{t, j} \geq \sqrt{B A} \\ \{\emptyset\}, & \text { otherwise. }\end{cases}
$$


The feasible regions of $\phi$, where $x_{2} \leq r$ and $x_{1} \leq r$, are the intersections of (78) and (91). As a result,

$$
\phi \in \begin{cases}{\left[\mathcal{F}_{2}, \pi-\mathcal{F}_{1}\right],} & \text { if } r^{2} A \geq B, l_{t, j} \geq \frac{r^{2} A+B}{2 r} \\ {\left[0, \pi-\mathcal{F}_{1}\right],} & \text { if } r^{2} A>B, \sqrt{B A} \leq l_{t, j}<\frac{r^{2} A+B}{2 r} \\ \{\emptyset\}, & \text { otherwise. }\end{cases}
$$

The feasible regions of $\phi$, where $x_{2} \leq r$ and $x_{1}>r$, are the intersections of (78) and (84). As a result,

$$
\phi \in \begin{cases}{\left[0, \mathcal{F}_{2}\right],} & \text { if } r^{2} A \leq B, \frac{r^{2} A+B}{2 r} \leq l_{t, j} \\ {\left[0, \mathcal{F}_{2}\right),} & \text { if } r^{2} A>B, \frac{r^{2} A+B}{2 r} \leq l_{t, j} \\ \{\emptyset\}, & \text { otherwise. }\end{cases}
$$

As a result, when $x_{2} \leq r$ and $x_{1} \leq r$, we have (31). Meanwhile, when $x_{2} \leq r$ and $x_{1}>r$, we have (32).

For $A<0, B>0$ : Comparing $x_{1}$ with 0 gives

$$
\phi \in[0, \pi], \text { for } x_{1}<0 ; \text { and } \phi=\emptyset, \text { for } x_{1} \geq 0 .
$$

Comparing $x_{2}$ with 0 gives

$$
\phi=\emptyset, \text { for } x_{2}<0 ; \text { and } \phi \in[0, \pi], \text { for } x_{2} \geq 0 .
$$

Comparing $x_{2}$ with $r$,

$$
x_{2}>r \Rightarrow \sqrt{\left(l_{t, j} \cos \phi\right)^{2}-B A}>l_{t, j} \cos \phi-r A .
$$

If $l_{t, j} \cos \phi-r A<0$, then

$$
\phi \in \begin{cases}\left(\mathcal{F}_{3}, \pi\right], & \text { if } l_{t, j} \geq-r A \\ \{\emptyset\}, & \text { if } l_{t, j}<-r A\end{cases}
$$

If

$$
l_{t, j} \cos \phi-r A \geq 0
$$

then

$$
\phi \in \begin{cases}{\left[0, \mathcal{F}_{3}\right],} & \text { if } l_{t, j} \geq-r A \\ {[0, \pi],} & \text { if } l_{t, j}<-r A .\end{cases}
$$

Assuming that (41) and (98) hold, solving (96) gives

$$
\phi \in \begin{cases}{[0, \pi],} & \text { if }-r^{2} A<B, l_{t, j}<\frac{r^{2} A+B}{2 r} \\ \left(\mathcal{F}_{2}, \pi\right], & \text { if }-r^{2} A \geq B, l_{t, j} \geq \frac{-r^{2} A-B}{2 r} \\ \left(\mathcal{F}_{2}, \pi\right], & \text { if }-r^{2} A<B, l_{t, j} \geq \frac{r^{2} A+B}{2 r} \\ \{\emptyset\}, & \text { otherwise. }\end{cases}
$$

Then, combining (99) and (100) with intersections yields

$$
\phi \in \begin{cases}{[0, \pi],} & \text { if }-A<\frac{B}{r^{2}},-A \geq \frac{B}{3 r^{2}}, l_{t, j}<\frac{r^{2} A+B}{2 r} \\ {[0, \pi],} & \text { if }-A<\frac{B}{r^{2}},-A<\frac{B}{3 r^{2}}, l_{t, j}<-r A \\ \left(\mathcal{F}_{2}, \pi\right], & \text { if }-A \geq \frac{B}{r^{2}}, \frac{-r^{2} A-B}{2 r} \leq l_{t, j}<-r A \\ \left(\mathcal{F}_{2}, \pi\right], & \text { if }-A<\frac{B}{r^{2}},-A>\frac{B}{3 r^{2}}, \frac{r^{2} A+B}{2 r} \leq l_{t, j}<-r A \\ {\left[0, \mathcal{F}_{3}\right],} & \text { if }-A<\frac{B}{r^{2}},-A<\frac{B}{3 r^{2}},-r A \leq l_{t, j}<\frac{r^{2} A+B}{2 r} \\ \left(\mathcal{F}_{2}, \mathcal{F}_{3}\right], & \text { if }-A \geq \frac{B}{r^{2}}, l_{t, j} \geq-r A \\ \left(\mathcal{F}_{2}, \mathcal{F}_{3}\right], & \text { if }-A<\frac{B}{r^{2}},-A \geq \frac{B}{3 r^{2}}, l_{t, j} \geq-r A \\ \left(\mathcal{F}_{2}, \mathcal{F}_{3}\right], & \text { if }-A<\frac{B}{r^{2}},-A<\frac{B}{3 r^{2}}, l_{t, j} \geq \frac{r^{2} A+B}{2 r} \\ \{\emptyset\}, & \text { otherwise. }\end{cases}
$$

The unions of (97) and (101) yield

$$
\phi \in \begin{cases}{[0, \pi],} & \text { if }-r^{2} A<B, l_{t, j}<\frac{r^{2} A+B}{2 r} \\ \left(\mathcal{F}_{2}, \pi\right], & \text { if } l_{t, j} \geq \frac{\left|r^{2} A+B\right|}{2 r} \\ \{\emptyset\}, & \text { otherwise. }\end{cases}
$$

Meanwhile,

$$
x_{2} \leq r \Rightarrow \sqrt{\left(l_{t, j} \cos \phi\right)^{2}-B A} \leq l_{t, j} \cos \phi-r A .
$$

If $l_{t, j} \cos \phi-r A \geq 0$, then

$$
\phi \in \begin{cases}{\left[0, \mathcal{F}_{3}\right],} & \text { if } l_{t, j} \geq-r A \\ {[0, \pi],} & \text { if } l_{t, j}<-r A\end{cases}
$$

Assuming that (41) hold, solving (103) gives

$$
\phi \in \begin{cases}{[0, \pi],} & \text { if }-r^{2} A>B, l_{t, j}<\frac{-r^{2} A-B}{2 r} \\ {\left[0, \mathcal{F}_{2}\right],} & \text { if } l_{t, j} \geq \frac{\left|-r^{2} A-B\right|}{2 r} \\ \{\emptyset\}, & \text { otherwise. }\end{cases}
$$

The intersections of (104) and (105) are given by (105). Hence, the feasible regions for $x_{2}>r$ or $0 \leq x_{2} \leq r$ are given by (102) and (105), respectively. As a result, when $x_{2}>r$, we have (33). Meanwhile, when $0 \leq x_{2} \leq r$, we have (34).

For $A>0, B<0$ : Comparing $x_{1}$ with 0 gives

$$
\phi \in[0, \pi], \text { for } x_{1} \geq 0 ; \text { and } \phi=\emptyset \text {, for } x_{1}<0 \text {. }
$$

Comparing $x_{2}$ with 0 gives

$$
\phi=\emptyset, \text { for } x_{2} \geq 0 ; \text { and } \phi \in[0, \pi] \text { for } x_{2}<0 .
$$

Comparing $x_{1}$ with $r$,

$$
x_{1}>r \Rightarrow \sqrt{\left(l_{t, j} \cos \phi\right)^{2}-B A}>r A-l_{t, j} \cos \phi .
$$

If $r A-l_{t, j} \cos \phi<0$, then

$$
\phi \in \begin{cases}{\left[0, \mathcal{F}_{3}\right),} & \text { if } l_{t, j} \geq r A \\ \{\emptyset\}, & \text { if } l_{t, j}<r A .\end{cases}
$$

If

$$
r A-l_{t, j} \cos \phi \geq 0
$$

then

$$
\phi \in \begin{cases}{\left[\mathcal{F}_{3}, \pi\right],} & \text { if } l_{t, j} \geq r A \\ {[0, \pi],} & \text { if } l_{t, j}<r A .\end{cases}
$$

Assuming that (41) and (110) hold, solving (108) gives

$$
\phi \in \begin{cases}{[0, \pi],} & \text { if }-r^{2} A>B, l_{t, j}<\frac{-r^{2} A-B}{2 r} \\ {\left[0, \mathcal{F}_{2}\right],} & \text { if }-r^{2} A \leq B, l_{t, j} \geq \frac{r^{2} A+B}{2 r} \\ {\left[0, \mathcal{F}_{2}\right],} & \text { if }-r^{2} A>B, l_{t, j} \geq \frac{-r^{2} A-B}{2 r} \\ \{\emptyset\}, & \text { otherwise. }\end{cases}
$$

Then, combining (111) and (112) with intersections yields

$$
\phi \in \begin{cases}{[0, \pi],} & \text { if }-A>\frac{B}{r^{2}},-A \geq \frac{B}{3 r^{2}}, l_{t, j}<r A \\ {[0, \pi],} & \text { if }-A>\frac{B}{r^{2}},-A<\frac{B}{3 r^{2}}, l_{t, j}<\frac{-r^{2} A-B}{2 r} \\ {\left[0, \mathcal{F}_{2}\right],} & \text { if }-A \leq \frac{B}{r^{2}}, \frac{r^{2} A+B}{2 r} \leq l_{t, j}<r A \\ {\left[0, \mathcal{F}_{2}\right],} & \text { if }-A>\frac{B}{r^{2}},-A<\frac{B}{3 r^{2}}, \frac{-r^{2} A-B}{2 r} \leq l_{t, j}<r A \\ {\left[\mathcal{F}_{3}, \pi\right],} & \text { if }-A>\frac{B}{r^{2}},-A>\frac{B}{3 r^{2}}, r A \leq l_{t, j}<\frac{-r^{2} A-B}{2 r} \\ {\left[\mathcal{F}_{3}, \mathcal{F}_{2}\right],} & \text { if }-A \leq \frac{B}{r^{2}}, l_{t, j} \geq r A \\ {\left[\mathcal{F}_{3}, \mathcal{F}_{2}\right],} & \text { if }-A>\frac{B}{r^{2}},-A \geq \frac{B}{3 r^{2}}, l_{t, j} \geq \frac{-r^{2} A-B}{2 r} \\ {\left[\mathcal{F}_{3}, \mathcal{F}_{2}\right],} & \text { if }-A>\frac{B}{r^{2}},-A<\frac{B}{3 r^{2}}, l_{t, j} \geq r A \\ \{\emptyset\}, & \text { otherwise. }\end{cases}
$$


The unions of (109) and (113) yield

$$
\phi \in \begin{cases}{[0, \pi],} & \text { if }-r^{2} A>B, l_{t, j}<\frac{-r^{2} A-B}{2 r} \\ {\left[0, \mathcal{F}_{2}\right],} & \text { if } l_{t, j} \geq \frac{\left|r^{2} A+B\right|}{2 r} \\ \{\emptyset\}, & \text { otherwise. }\end{cases}
$$

Meanwhile,

$$
x_{1} \leq r \Rightarrow \sqrt{\left(l_{t, j} \cos \phi\right)^{2}-B A} \leq r A-l_{t, j} \cos \phi .
$$

If

$$
r A-l_{t, j} \cos \phi \geq 0
$$

then

$$
\phi \in \begin{cases}{\left[\mathcal{F}_{3}, \pi\right],} & \text { if } l_{t, j} \geq r A \\ {[0, \pi],} & \text { if } l_{t, j}<r A .\end{cases}
$$

Assuming that (41) and (116) hold, solving (115) gives

$$
\phi \in \begin{cases}{[0, \pi],} & \text { if }-r^{2} A<B, l_{t, j}<\frac{r^{2} A+B}{2 r} \\ {\left[\mathcal{F}_{2}, \pi\right],} & \text { if } l_{t, j} \geq \frac{\left|r^{2} A+B\right|}{2 r} \\ \{\emptyset\}, & \text { otherwise. }\end{cases}
$$

The intersections of (117) and (118) is (118). Hence, the feasible regions for $x_{1}>r$ or $0 \leq x_{1} \leq r$ are given by (114) and (118), respectively. As a result of the above discussions, when $x_{1}>r$, we have (35). Meanwhile, when $0 \leq x_{1} \leq r$, we have (36).

For $A=0$ : The corresponding solution to $1+$ $\frac{\left(H^{2}+l_{t, e}^{2}\right)^{\frac{-\alpha}{2}} \eta P_{s}}{\left(l_{t, j}^{2}+l_{t, e}^{2}-2 l_{t, j} l_{t, e} \cos (\phi)\right)^{\frac{-\alpha}{2}} P_{j}} \leq y$ is given by $\cos \phi \geq \frac{l_{t, j}^{2}-H^{2}}{2 l_{t, j} l_{t, e}}$. Thus, we have

$$
\phi \in \begin{cases}{\left[0, \cos ^{-1}\left(\frac{l_{t, j}^{2}-H^{2}}{2 l_{t, j} l_{t, e}}\right)\right],} & \text { if } \frac{\left|l_{t, j}^{2}-H^{2}\right|}{2 l_{t, j}} \leq l_{t, e} \\ {[0, \pi],} & \text { if } \frac{-l_{t, j}^{2}+H^{2}}{2 l_{t, j}}>l_{t, e} \\ \{\emptyset\}, & \text { otherwise. }\end{cases}
$$

Comparing $l_{t, e}$ from (119) with $r$, we have (37) - (39).

\section{REFERENCES}

[1] M. Mozaffari, W. Saad, M. Bennis, Y.-H. Nam, and M. Debbah, "A tutorial on UAVs for wireless networks: Applications, challenges, and open problems," IEEE Commun. Surveys Tuts, to be published.

[2] H. Kim, L. Mokdad, and J. Ben-Othman, "Designing UAV surveillance frameworks for smart city and extensive ocean with differential perspectives," IEEE Commun. Mag., vol. 56, no. 4, pp. 98-104, Apr. 2018.

[3] S. A. R. Naqvi, S. A. Hassan, H. Pervaiz, and Q. Ni, "Drone-aided communication as a key enabler for $5 \mathrm{G}$ and resilient public safety networks," IEEE Commun. Mag., vol. 56, no. 1, pp. 36-42, Jan. 2018.

[4] Y. Zhu, G. Zheng, and M. Fitch, "Secrecy rate analysis of UAV-enabled mmwave networks using Matérn hardcore point processes," IEEE J. Sel. Areas Commun., vol. 36, no. 7, pp. 1397-1409, July 2018.

[5] V. Chang, P. Chundury, and M. Chetty, "Spiders in the sky: User perceptions of drones, privacy, and security," in Proce. CHI Conf. Human Factors Comput. Syst. Denver, CO: ACM, 2017, pp. 6765-6776.

[6] W. K. Harrison, J. Almeida, M. R. Bloch, S. W. McLaughlin, and J. Barros, "Coding for secrecy: An overview of error-control coding techniques for physical-layer security," IEEE Signal Process. Mag., vol. 30, no. 5, pp. 41-50, Sept. 2013.

[7] G. Chen and J. P. Coon, "Secrecy outage analysis in random wireless networks with antenna selection and user ordering," IEEE Wireless Commun. Lett., vol. 6, no. 3, pp. 334-337, June 2017.

[8] H. V. Poor and R. F. Schaefer, "Wireless physical layer security," Proc. National Academy of Sciences, vol. 114, no. 1, pp. 19-26, Jan. 2017.

[9] J. Yao, S. Feng, X. Zhou, and Y. Liu, "Secure routing in multihop wireless ad-hoc networks with decode-and-forward relaying," IEEE Trans. Commun., vol. 64, no. 2, pp. 753-764, Feb. 2016.
[10] P. C. Pinto, J. Barros, and M. Z. Win, "Secure communication in stochastic wireless networks-Part II: Maximum rate and collusion," IEEE Trans. Inf. Forensics Security, vol. 7, no. 1, pp. 139-147, Feb. 2012.

[11] G. Chen, J. P. Coon, and M. Di Renzo, "Secrecy outage analysis for downlink transmissions in the presence of randomly located eavesdroppers," IEEE Trans. Inf. Forensics Security, vol. 12, no. 5, pp. 1195-1206, May 2017.

[12] J. Tang, G. Chen, and J. P. Coon, "Meta distribution of the secrecy rate in the presence of randomly located eavesdroppers," IEEE Wireless Commun. Lett., vol. 7, no. 4, pp. 630-633, Aug. 2018.

[13] C. Zhang and W. Zhang, "Spectrum sharing for drone networks," IEEE J. Sel. Areas Commun., vol. 35, no. 1, pp. 136-144, Jan. 2017.

[14] V. V. Chetlur and H. S. Dhillon, "Downlink coverage analysis for a finite 3D wireless network of unmanned aerial vehicles," IEEE Trans. Commun., vol. 65, no. 10, pp. 4543-4558, Oct. 2017.

[15] ITU-R, "Propagation data and prediction methods required for the design of terrestrial broadband radio access systems operating in a frequency range from 3 to $60 \mathrm{GHz}$," ITU-R, Recommendation P.1410-5, Feb. 2012. [Online]. Available: https://www.itu.int/dms_pubrec/itu-r/rec/p/RREC-P.1410-5-201202-I!!PDF-E.pdf [Accessed: Apr. 05, 2019]

[16] A. Al-Hourani, S. Kandeepan, and S. Lardner, "Optimal LAP altitude for maximum coverage," IEEE Wireless Commun. Lett., vol. 3, no. 6, pp. 569-572, Dec. 2014.

[17] Q. Wang, Z. Chen, W. Mei, and J. Fang, "Improving physical layer security using UAV-enabled mobile relaying," IEEE Wireless Commun. Lett., vol. 6, no. 3, pp. 310-313, June 2017.

[18] M. Cui, G. Zhang, Q. Wu, and D. W. K. Ng, "Robust trajectory and transmit power design for secure UAV communications," IEEE Trans. Veh. Technol., vol. 67, no. 9, pp. 9042-9046, Sept. 2018.

[19] G. Zhang, Q. Wu, M. Cui, and R. Zhang, "Securing UAV communications via joint trajectory and power control," IEEE Trans. Wireless Commun., vol. 18, no. 2, pp. 1376 - 1389, 2019.

[20] Y. Zhou, P. L. Yeoh, H. Chen, Y. Li, R. Schober, L. Zhuo, and B. Vucetic, "Improving physical layer security via a UAV friendly jammer for unknown eavesdropper location," IEEE Trans. Vehicular Techn., vol. 67, no. 11, pp. $11280-11284$, Nov. 2018.

[21] A. Li, Q. Wu, and R. Zhang, "UAV-enabled cooperative jamming for improving secrecy of ground wiretap channel," IEEE Wireless Commun. Lett., vol. 32, no. 2, pp. 286-289, Feb. 2019.

[22] Holland Michel, Arthur, "Counter-drone systems," Center for the Study of the Drone at Bard College, Tech. Rep., Feb. 2018. [Online]. Available: http://dronecenter.bard.edu/counter-drone-systems/ [Accessed: Apr. 05, 2019]

[23] M. Mozaffari, W. Saad, M. Bennis, and M. Debbah, "Unmanned aerial vehicle with underlaid device-to-device communications: Performance and tradeoffs," IEEE Trans. Wireless Commun., vol. 15, no. 6, pp. 3949-3963, June 2016.

[24] D. Tse and P. Viswanath, Fundamentals of wireless communication. Cambridge, UK: Cambridge University Press, 2005.

[25] G. Chen, Y. Gong, P. Xiao, and J. A. Chambers, "Physical layer network security in the full-duplex relay system," IEEE Trans. Inf. Forensics Security, vol. 10, no. 3, pp. 574-583, Mar. 2015.

[26] G. Chen, Y. Gong, P. Xiao, and J. A. Chambers, "Dual antenna selection in secure cognitive radio networks," IEEE Tran. Veh. Technol., vol. 65, no. 10, pp. 7993-8002, Oct. 2016.

[27] A. Blank, R. Kastner, and H. Levanon, "Exploring new active materials for low-noise room-temperature microwave amplifiers and other devices," IEEE Trans. Microw. Theory Tech., vol. 46, no. 12, pp. 2137-2144, Dec. 1998.

[28] J. L. Alford and M. Thomas, "Thermoelectric cooling of low-noise amplifier transistors in wireless communications networks," Nov. 152005 , US Patent 6,965,515.

[29] S. Weinreb, J. C. Bardin, and H. Mani, "Design of cryogenic SiGe lownoise amplifiers," IEEE Trans. Microw. Theory Tech., vol. 55, no. 11, pp. 2306-2312, Nov. 2007.

[30] "NIST Digital Library of Mathematical Functions," F. W. J. Olver, A. B. Olde Daalhuis, D. W. Lozier, B. I. Schneider, R. F. Boisvert, C. W. Clark, B. R. Miller and B. V. Saunders, eds. [Online]. Available: http://dlmf.nist.gov/ [Accessed: Apr. 05, 2019]

[31] J. Tang, G. Chen, J. P. Coon, and D. E. Simmons, "Distance distributions for Matérn cluster processes with application to network performance analysis," in Proc. IEEE ICC, Paris, France, May 2017. 


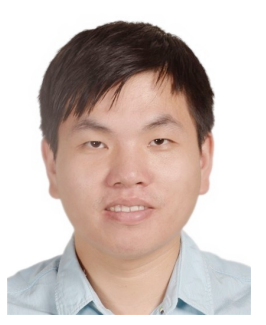

Jinchuan Tang (S'17) received his B.Eng. degree in electronic information engineering from Chongqing University of Posts and Telecommunications in 2011. He worked as an Assistant Engineer in Huawei Technologies Co., Ltd. from 2011 to 2012. He obtained the M.Sc. degree with Distinction in wireless communications from the University of Southampton in early 2014. He is currently pursuing a DPhil degree under the supervision of Professor Justin P. Coon with the Department of Engineering Science, University of Oxford. His current research interests include optimum power allocation, random geometric networks, secure communication, and route selection in wireless networks.

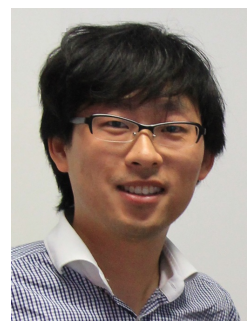

Gaojie Chen (S'09 - M'12 - SM'18) received the B.Eng. and B.Ec. degrees in electrical information engineering and international economics and trade from Northwest University, China, in 2006, and the M.Sc. (Hons.) and Ph.D. degrees in electrical and electronic engineering from Loughborough University, Loughborough, U.K., in 2008 and 2012, respectively. From 2008 to 2009, he was a Software Engineering with DTmobile, Beijing, China, and from 2012 to 2013, he was a Research Associate with the School of Electronic, Electrical and Systems Engineering, Loughborough University. He was a Research Fellow with 5GIC, Faculty of Engineering and Physical Sciences, University of Surrey, U.K., from 2014 to 2015. Then he was a Research Associate with the Department of Engineering Science, University of Oxford, U.K., from 2015 to 2018 . He is currently a Lecturer with the Department of Engineering, University of Leicester, U.K. His research interests include information theory, wireless communications, IoT, cognitive radio, secrecy communication and random geometric networks $\mathrm{He}$ received the Exemplary Reviewer Certificate of the IEEE WIRELESS COMMUNICATION LETTERS in 2018. He currently serves as an Editor of the IET ELECTRONICS LETTERS.

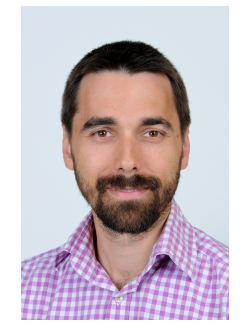

Justin P. Coon (S'02 - M'05 - SM'10) received a BSc. degree (with distinction) in electrical engineering from the Calhoun Honours College, Clemson University, USA and a Ph.D in communications from the University of Bristol, U.K. in 2000 and 2005, respectively. In 2004, he joined Toshiba Research Europe Ltd. (TREL) as a Research Engineer working in its Bristol based Telecommunications Research Laboratory (TRL), where he conducted research on a broad range of communication technologies and theories, including single and multi-carrier modulation techniques, estimation and detection, diversity methods, system performance analysis and networks. He held the position of Research Manager from 20102013, during which time he led all theoretical and applied research on the physical layer at TRL. Dr Coon was a Visiting Fellow with the School of Mathematics at the University of Bristol from 2010-2012, and held a position as Reader in the Department of Electrical and Electronic Engineering at the same university from 2012-2013. He joined the University of Oxford in 2013 where he is currently an Associate Professor with the Department of Engineering Science and a Tutorial Fellow of Oriel College.

Dr Coon is the recipient of TRLs Distinguished Research Award for his work on block-spread CDMA, aspects of which have been adopted as mandatory features in the 3GPP LTE Rel-8 standard. He is also a co-recipient of two 'best paper' awards for work presented at ISWCS 13 and EuCNC 14. Dr Coon has published in excess of 150 papers in leading international journals and conferences, and is a named inventor on more than 30 patents. He served as an Editor for IEEE TRANSACTIONS ON WIRELESS COMMUNICATIONS (2007 2013), IEEE TRANSACTIONS ON VehicUlar TECHNOLOGY (2013 - 2016), IEEE WiRELESS COMMUNiCATIONS LETTERS (2016 - present) and IEEE COMmunications LetTers (2017 - present). Dr Coons research interests include communication theory, information theory and network theory. 